\title{
Diversity Management Instruments in German Organizations: Effectiveness and Preferences from Corporate and Employee Perspectives
}

\author{
Jens Landwehr \\ Faculty of Management, Economics and Social Sciences, Seminar of Personnel Economics and HRM, University of Cologne, Cologne, \\ Germany
}

\section{Email address:}

Jenslandwehr@me.com

\section{To cite this article:}

Jens Landwehr. Diversity Management Instruments in German Organizations: Effectiveness and Preferences from Corporate and Employee Perspectives. Journal of Human Resource Management. Vol. 4, No. 6, 2016, pp. 100-123. doi: 10.11648/j.jhrm.20160406.13

Received: November 25, 2016; Accepted: December 6, 2016; Published: January 24, 2017

\begin{abstract}
While diversity management appears to rank high on the HR agenda of many organizations in Germany, both status and quality of diversity management in Germany remain lower than in most European countries, and by far lower than in the US. This paper discusses the relevance organizations in Germany attach to the topic of diversity, identifies the measures actually employed, and shows how effective these measures are in practice. Based on the responses of 73 organizations and 285 employees, the study empirically measures the use and effectiveness of diversity measures and identifies differences from the perspective of the organization versus the employee. In the area of Gender diversity companies employ the largest breadth of measures that also appear very effective. In the areas of Age and Ethnic diversity, companies tend to employ much fewer measures which also don't appear to be the most effective, showing less relevance and experience with relevant instruments. We also found that measures considered by employees to be most effective often differ from the measures used in practice and prioritized by HR professionals. In general, from an employee perspective, diversity measures appear to be connected to better career and development opportunities. Companies in Germany largely follow the diversity measures prescribed by the state without using their diversity profile for differentiation in employer branding. The results of the study give an overview of the diversity measures in use today in German organizations and of their effectiveness to achieve diversity objectives.
\end{abstract}

Keywords: Personality Assessment, Performance Management, Talent Management, Personality, Potential, Personality Talent Management, Potential, Social Responsibility

\section{Introduction}

\subsection{Definitions of Diversity}

In recent years, diversity management has become a widely discussed topic both in the political arena and society at large. The fundamental objective pursued by organizations with their diversity management efforts is to develop a "diverse" employee structure. In this context, "diversity" can relate to several employee attributes (gender, age, ethnic origin, religion, sexual orientation and physical abilities). Although there are large cultural differences in the interpretation of the term diversity, working definitions in the US and the UK agree on the typical motivations behind this objective are closely intertwined with issues of demographic change and the resulting lack of qualified expert and management personnel (Nkomo \& Cox 1996; Kandola \& Fullerton, 1998; Arrendondo, 1996). In consequence, improving one's attractiveness as an employer and one's capability to recruit talents is therefore one of the primary goals of the diversity management efforts undertaken by organizations. In addition, using the different perspectives contributed by diverse employees helps to foster the innovative ability and motivation of employees, develop the corporate culture, successfully open up new markets and address diverse consumer groups.

The term diversity management can be traced back to the publication of a report in 1987 titled Workforce 2000 (Johnson et al. 1987). Diversity management has its roots in the Equal Employment Opportunity (EEO) and Affirmative Action (AA) 
movements in the US and can be traced to the publication of a report in 1987 titled Workforce 2000 (Johnson et al., 1987) by the Hudson institute. This report projected demographic shifts leading up to the year 2000 in which the US workforce would dramatically diversify. Since then, the topic has been categorized by scholars as evolving (Thomas 1995), the stage of EEO/AA having followed by a 'Valueing Differences' phase, followed by a Managing Diversity Phase and finally entering the 'Global Diversity Management', a term mostly described by Ozbilgrin \& Tatli (2008).

\subsection{Problematization of Diversity Management Globally}

Diversity management has become a topic that ranks high on the HR agendas in many organizations. However, similar to the widespread and deep confusion around the equally fashionable HR area of "talent management" (Lewis \& Heckman 2006; Collings \& Mellahi, 2009), there are high levels of ambiguity and insecurity about how to appropriately manage diversity, about the objectives of diversity management, as well as the effectiveness of specific measures (Lorbiecki \& Jack, 2000), most notably the gender quota for women in leadership positions. It is not a new development that both cause and effect of diversity management is being discussed controversially. Rooted in the US Civil Rights movement, the creation of equal opportunities in organization has been a political topic from the start. Since its inception the 1980s, there has been a debate on whether diversity management should benefit the people or the organization. Against this background, a large scale problematization of diversity management has taken place through the different phases and in the broader social context (Thomas 1995), both in theory and in practice. The main aspects of criticism include: Control mechanism at play, which have recently been described by Costea \& Introna (2008); appropriate definitions of difference as being described by Kersten (2000), as well neo-liberal concerns voiced by Humphries \& Gries (1995).

Diversity management has been discussed controversially for the last 30 years from different perspectives. At the origin of the debate was the demographic argument, based on the assumption that a more diverse workforce will substitute the traditional white, heterosexual, married, career minded, male workforce. The discussion also has a political dimension (quota) as well as an economic argument. Rooted in the affirmative action movement in the US, diversity management has undergone several phases of ambivalence. Initially triggered though expected demographic change, the ambivalence also underwent a political phase, as well as the economic phase. The literature - originally very positivistic turned more critical when problems were encountered in the implementation of diversity management (Lorbiecki \& Jack, 2000). For example, diversity management was viewed as an instrument or tool that uses people's diversity as the means of achieving economic success based on control (Vince \& Booth, 1996). Garnsey \& Rees (1996) suggested that, instead of creating an atmosphere of tolerance and respect at the workplace, diversity management interventions appear to have triggered responses of antagonism and resentment on part of the 'managed diverse'. The critical perspective on diversity management has been reinforced by studies of practical examples in which diversity management has been misguided and abused to obstruct marking measures towards minorities (Subeliani \& Tsogas, 2005).

Over the years and the different phases of diversity management have also been critical voices on specific programs and measures. The criticism includes, that programs often perpetuate rather than combat inequalities, diminish the legacy of discrimination and continue to prescribe essentialist categories of difference. In addition, it has been noted that the focus is too often on differences rather than on inclusion.

Furthermore, there has been widespread unease about the objectives of diversity management. Is diversity about increasing the share of women in management positions, in order to comply with legal requirements? Is it about creating a diversity culture which is presumed to have a positive impact on the performance of the workforce as a result of more respectful cooperation of all diversity groups (e.g. employees with a migration background or mature employees)? Is diversity management rather a leadership issue? Is it an HR tool to make the organization more attractive as an employer? In any case, there is hardly any department or function head in German corporations who would not occupy himself or herself with this topic.

This widespread ambivalence in the assessment of the means and ends of diversity management is therefore a theme that can be traced through the history and different phases of diversity management, both internationally and in Germany. However, there are also some large differences between nations both in the practice and theory of diversity management. The differences are the result of real world variations in the need for diversity categories and the ensuing social and economic debate, as well as the use and prioritization of specific measures (Jonsen et al, 2013). This embeddedness in a social and political context makes it, however, also more complicated to compare study results across nations. As a result, any measurement of success of specific diversity measures has to be evaluated within its business, social and cultural context.

\subsection{The Limits of Diversity Management in Germany}

In comparison to the US, which have a tradition of a strong contextual embeddedness of diversity management in the managerial, economic and social discourse (McDonald, 2010), Germany has a relatively short diversity history. In comparison to other countries, the efforts of diversity management have been labeled 'Integrationism', historically mostly directed at the assimilation of Turks to the German work space. As a result, the history and prevalence of other diversity categories, especially geared towards ethnic diversity in general is relatively low. In Germany, in contrast to the US and UK, the phases described by cannot be extracted in the same way due to a lower level of maturity in the social and economic discourse (Tatli et al, 2012). 
Another clear indicator for a relatively low diversity maturity in Germany is that in organizations lower levels of diversity measures are being applied, and on top there is a tendency to apply measure with lower levels of effectiveness (Köppel, Ya, Lüdicke, 2007).

In Germany, the typical objectives of diversity management are closely intertwined with the demographic change and the resulting shortage of professional and management personnel. For the companies, enhancing their attractiveness as an employer and attracting new talents are primary objectives. In addition, having diverse perspectives in the workforce promotes the innovative power and motivation of the employees and thereby makes a positive contribution to the corporate culture and helps to successfully tap new markets and consumer groups. Therefore, in recent years, the topic of gender diversity has become a widely discussed topic in the political arena and in society at large. Since that time, and spurred by state regulation, companies in Germany aim at developing and shaping a "diverse" workforce, spurred by the strong media debate around this issue and additional possible regulative influences with respect to "Gender diversity".

However across all other diversity categories, and despite the ongoing debate on increasing the number of women in management positions, German organizations employ much lower levels of diversity measures compared to other countries. This is surprising as numbers of migrant workers in their domestic operations continually rise, as well as domestic workers sent abroad, and international business relations are continuously on the rise and have outreached those of traditional immigrant countries such as the US and UK. In addition, employees' and job applicants' demands especially in Germany increase. Given the demographic change and the intense competition in the labor market for highly qualified employees, the prevailing attitude generally appears to be that diversity management is of great importance for a successful future of the company. However, high importance is not the same as effectiveness, and a critical debate is happening to a very limited extend.

In addition, diversity measures are implemented to a lesser degree in German organizations as compared to Europe as a whole and the US/UK. Overall, German organizations don't apply enough diversity measures and the measures implied appear to be less effective compared to other countries, and they also don't apply the measures correctly.

As a result, German organizations are in some ways less well prepared for the challenges associated with the demographic change compared to companies in other comparable European countries, which could become a disadvantage as the war for talent becomes even more international. Even today, a large gap can also be identified with smaller countries more adapt to immigrant culture such as the Netherlands and Denmark. For example, Royal Dutch Shell and British Petroleum employ higher numbers of international stuff in leadership positions than do comparable German companies (insert quote). Overall, the orientation of diversity management in Germany appears to be less mature.
As in other nations, diversity in Germany has followed a path-dependent trajectory in which the meaning of diversity management has been bent, stretched, shrunk, and fixed to fit the national traditions and requirements of social and economic activity. While in the US a bottom line orientation dominates, in France the debate has a moral undertone, and in the UK a social regulation dominates, is diversity in Germany embedded around integration topics within the German business and cultural context (Tatli et al, 2012). However, this is a limitation of the opportunities of diversity management both for organizations and society (Krell 2008) and most researchers agree that Germany would benefit from additional diversity measures. Therefore, this research can be understood as a component towards an integrated relational theory of diversity management.

\subsection{Measuring the Effectiveness of Diversity Management in Practice}

In addition to the diversity lag in practice, there is also a clear lag in the critical theoretical review of diversity in Germany. In the last years quite a number of studies have been carried out in Germany on diversity management (Köppel et al, 2007). However, these studies have mostly been descriptive in nature and only a few addressed the actual design and effectiveness of specific actions taken to enhance diversity (Süss \& Kleiner, 2005). In addition, these studies neither have been aimed at establishing a link between the use of certain diversity measures and the resulting diversity culture in specific German organizations, nor how effective specific instruments are from an employer and an employee perspective. Only a few of these studies have addressed the specific design of the diversity measures taken in organizations and the actual effectiveness of specific measures (Stuber, 2004). Actual measures employed and their effects remain elusive. In addition, German literature on diversity management omits large parts of aspects of problematization of diversity management as predominant in the US and other European cultures. This it is no surprise, that according to a number of studies, Germany lags behind in applying diversity measures (Köppel et al, 2007). The study therefore intends to provide insights into the prioritization, workings and effectiveness of specific measures and an explanation why diversity management is behind in Germany in many ways. Our study focuses on the importance attached by organizations to diversity management, on how diversity management is integrated in the organizational structure and implemented in company policies, on which and to what degree specific instruments are practically used to promote the three most common diversity aspects - gender, age and ethnic diversity.

However, our study was not limited to asking employers for their perspective. So, what precisely are the objectives organizations pursue with diversity management, and how well are these objectives supported by the multitude of diversity instruments and programs that are currently in place in German organizations? Is diversity management suitable to make an organization more successful? We would like to 
find out which instruments are success critical with respect to which objectives. How do you measure the success of diversity management and what are the influencing factors determine, how effective diversity measures are? Is it national culture, company culture, or personality of the employees? Measuring the success of diversity measures is not trivial as it needs to include the contextual aspects. Is it the economic success, is it employee satisfaction? In addition, as described above, every country has different aspect of diversity due to culture link. But which instruments are success critical with respect to which objectives? Very little research has been done around these questions. In light of the contextual orientation of diversity measures, cross national studies of diversity measures have been very limited. But then its needs to include contextual measures as well.

Our study therefore focuses on the significance of diversity management from the point of view of the company, the organizational responsibilities for and integration of the topic into HR and corporate policies, and the degree to which instruments to promote gender, age and ethnic diversity are actually being used. The key question is what determines whether measures are success critical, i.e. is it the measures itself, is it the employees who determine the success of the diversity measures, or the culture of the organization.

\section{Research Questions}

The aim of this explorative study is to empirically establish a link between the use of certain instruments and certain qualitative and quantitative ends. Quantitative ends include the quota of women in management, the percentage of employees older than 56 years, and the percentage of international (non-German) staff. Qualitative ends include the perception of diversity and discrimination in particular organizations, value acknowledgement, personal commitment to the organization, work-family conflict, personal attitudes towards care and performance, and personal career preferences. We expected positive relationships between the use of certain instruments and these ends. In order to clarify the linkages between, we will draw separate hypotheses for different diversity instruments, including gender, age and ethnic diversity.

Initially we measure today's weight of diversity management in German organizations and establish a complete overview of the prevalence of specific instruments. We also measured the organizational infrastructure, and size of departments as well as integration in the wider range of personnel measure as well as corporate policy.

Two sets of success metrics were used. To identify success critical instruments to support gender equality, we used the quota of women in management. To identify success critical instruments to more age diversity, we used the quota of 5665-year employees. To identify success critical ethnic diversity instruments the rate of non-German staff was used; instead, employees were likewise asked for their perception of the importance and effectiveness of various diversity management instruments.
In order to back the results by the employee perspective, in addition employees of the organizations participating in the survey were asked to identify instruments in their organizations, as well as the ends. Thus it was possible to establish convincing success criteria for instruments of diversity.

Furthermore, the aim of the study is to compare assessments from HR professionals, objective success measures, as well as from employees.

\section{Hypotheses}

Hypothesis 1a: Diversity measures still have a relatively low urgency and are not wide spread in German organizations (especially Age and Ethnic diversity); However, German organizations expect the importance to grow significantly in the future.

Hypothesis 1b: The diversity measures offered by German organizations differ from the one prioritized as most effective by experts.

Hypothesis 1c: Employees rate different measures high compared to the measures actually offered.

Hypothesis 2: In terms of Gender diversity instruments, companies are not offering sufficient levels of qualitative instruments such as Job sharing, gender quotas, mentoring and corporate childcare although these are effective and desired my employees.

Hypothesis 3: In terms of Age diversity instruments, companies are not offering sufficient levels of systematic personnel development, age consistent content, horizontal moves and age mixed teams although these are effective and desired my employees.

Hypothesis 4: In terms of Ethnic diversity instruments, companies are not attracting well enough qualified talent with international experience although these are effective and desired my employees.

Hypothesis 5: Diversity culture is more relevant as a success factor as employee personality.

\section{Method}

\subsection{Sample}

On the basis of participant data from a survey conducted in 2009,600 of Germany's largest organizations were contacted in Winter/Spring 2011/2012. The invitation to participate was addressed to the Personnel Managers/departments by email. The broad purpose was explained to the participants combined with the incentive to receive the study results upon completion of the study as well as a free benchmarking against the other participants. As a result, the online questionnaire consisting of 52 questions was completed by 73 personnel managers or talent and diversity professionals. The average company size was about 22,800 .

In order to generate an overview over the employee perspective, employees of the participating companies were identified via Xing and contacted with the request to respond to an online questionnaire consisting of additional 52 
questions. This questionnaire was completed by 287 employees of 23 (out of the 73 participating) companies. As a result, we could run correlations between talent and diversity instruments as well as.

\subsection{Design and Procedure}

In the first part of the questionnaire, HR experts were asked to assess the relevance of diversity management to their organizations as well as provide some insights about the diversity management infrastructure (table 1a). In parallel, the first part of the employee questionnaire, participants were asked about their Perception of diversity and talent management from an employee perspective (table 1b). The second part in both the employer and employees questionnaires provided an overview over the prevalence of diversity management instruments in their respective organization. Interestingly, employer and employee perception also varied in this respect. Moreover there was also some significant variation in the effectiveness of instruments.

Finally, the employer sure asked for a broad variation of diversity related metrics (ends) as well as some sociographic date of the participating organizations. In a similar way, the employer survey asked for some sociographic date as well as some personality related self-assessment.

\section{Measures}

\subsection{Use of Instruments}

Participants were asked to assess the use of 32 instruments by diversity group (gender, age and ethnic) on a scale from not available to widely available. In addition, HR experts were asked to choose the instruments which are most effective from their perspective. Similarly, employees were asked about the use of instruments in their respective organizations.

\subsection{Effectiveness of Diversity Management}

We defined three measure of success for diversity measures, these include Quota (percentage) of women in leadership positions, Even age distribution, and Employees with non-German background.

In order to get an inclination for which instruments are linked to organizations we regressed the use of specific instruments with quotes of higher and low measures of success. Off course we understand that the logic is difficult.

Employee perception on the value of various objectives of diversity management, i.e.

- discrimination in the organization

- career and development opportunities

- recognition

- retention and personal commitment

- work family conflict

- personal perception on career and performance

- personal preferences on career development

- talent culture construct

\section{Results}

Increasing relevance of diversity management but little breadth and low levels of organization.

Table 1a provides an overview over the relevance of diversity and talent management in the context of German organizations. While the mean significance is as 3.25 at the middle range (sd 1.08446), the expected relevance for the future is expected to be much higher at 4.13 ( $\mathrm{sd} 0.53879)$.

Table 1a. Perception of diversity and talent management from an employer's perspective.

\begin{tabular}{|c|c|c|c|c|c|}
\hline & Variable code & Mean & Standard deviation & Range & Cronbach's alpha \\
\hline \multicolumn{6}{|l|}{ Relevance of Diversity Management } \\
\hline $\begin{array}{l}\text { How do you rank the significance of diversity management in your } \\
\text { organization? }\end{array}$ & Stellw & 3.25 & 1.084461 & $1-5$ & \multirow[t]{11}{*}{0,52} \\
\hline How will it change in the coming years? & veraend_st $\sim \mathrm{W}$ & 4.138889 & .5387925 & $3-5$ & \\
\hline Is there a main responsible for diversity management? & Beauftragter & .3239437 & .4713097 & $0-1$ & \\
\hline For how many years? & hre_beauf & 6.333333 & 4.809712 & $0-18$ & \\
\hline $\begin{array}{l}\text { Is there a position/department dealing mainly with diversity } \\
\text { management? }\end{array}$ & Abteilung & .6818182 & .4767313 & $0-1$ & \\
\hline How many employees & ma_abteilung & 6.166667 & 4.407294 & $0-16$ & \\
\hline Do you have concrete diversity goals for gender? & geschl_div $\sim$ S & .7538462 & .4341216 & $0-1$ & \\
\hline Do you have concrete diversity goals for age? & alters_div $\sim \mathrm{S}$ & .453125 & .5017331 & $0-1$ & \\
\hline Do you have concrete diversity goals for ethnic? & eth_divers & .3809524 & .4895215 & $0-1$ & \\
\hline Are diversity goals linked with personnel strategy & divers_per $\sim \mathrm{t}$ & .5671642 & .4992079 & $0-1$ & \\
\hline Are diversity goals linked with corporate strategy? & divers_all t & .3846154 & .4902903 & $0-1$ & \\
\hline \multicolumn{6}{|l|}{ Relevance of Talent Management } \\
\hline How will it change in the coming years? & vernd_stew_tm & 4.234375 & .5561257 & $2-5$ & \multirow{6}{*}{0,63} \\
\hline Is there a main responsible for talent management? & hauptveran $\sim \mathrm{m}$ & .78125 & .4166667 & $0-1$ & \\
\hline For how many years? & hre_hauptb $\sim \mathrm{m}$ & 7.6 & 15.54778 & $0-100$ & \\
\hline $\begin{array}{l}\text { Is there a position/department dealing mainly with talent } \\
\text { management? }\end{array}$ & eigene_abt $\sim \mathrm{m}$ & .78 & .418452 & $0-1$ & \\
\hline Staff in this department & ma_tm_abt & 9.633333 & 14.31778 & $1-80$ & \\
\hline Relevance of TM for employer branding & employer_b $\sim \mathrm{d}$ & 3.78125 & 1.105093 & $1-5$ & \\
\hline
\end{tabular}




\begin{tabular}{|c|c|c|c|c|c|}
\hline & Variable code & Mean & Standard deviation & Range & Cronbach's alpha \\
\hline Relevance of TM for university marketing & hs_marketing & 3.8125 & 1.12511 & $1-5$ & \\
\hline Relevance of TM for talent identification & ident_inte e & 4.203125 & .9116475 & $3-5$ & \\
\hline Relevance of TM for personnel development of internal talents & peronalent $\sim \mathrm{r}$ & 4.111111 & .9351748 & $2-5$ & \\
\hline Relevance of TM for succession management & Nachfolgepl & 3.84375 & 1.184808 & $1-5$ & \\
\hline Relevance other factors & Weitere & 3.545455 & 1.439697 & $1-5$ & \\
\hline Talent management goals anchored in personnel strategy & $\mathrm{tm} \_z i e l e \_\mathrm{p} \sim \mathrm{t}$ & .8064516 & .3983042 & $0-1$ & \\
\hline
\end{tabular}

At 0.7538 , diversity goals for gender are much higher than for age and ethnic diversity.

In contract to diversity management, means significance of talent management is ranked at 3.77 significantly higher than diversity management, and the expected relevance is expected also to be higher at 4.23 (sd).

Table $1 \mathrm{~b}$ provides an overview over diversity related perceptions from an employee perspective.

Table $1 \mathrm{~b}$. Perception of diversity and talent management from an employee perspective.

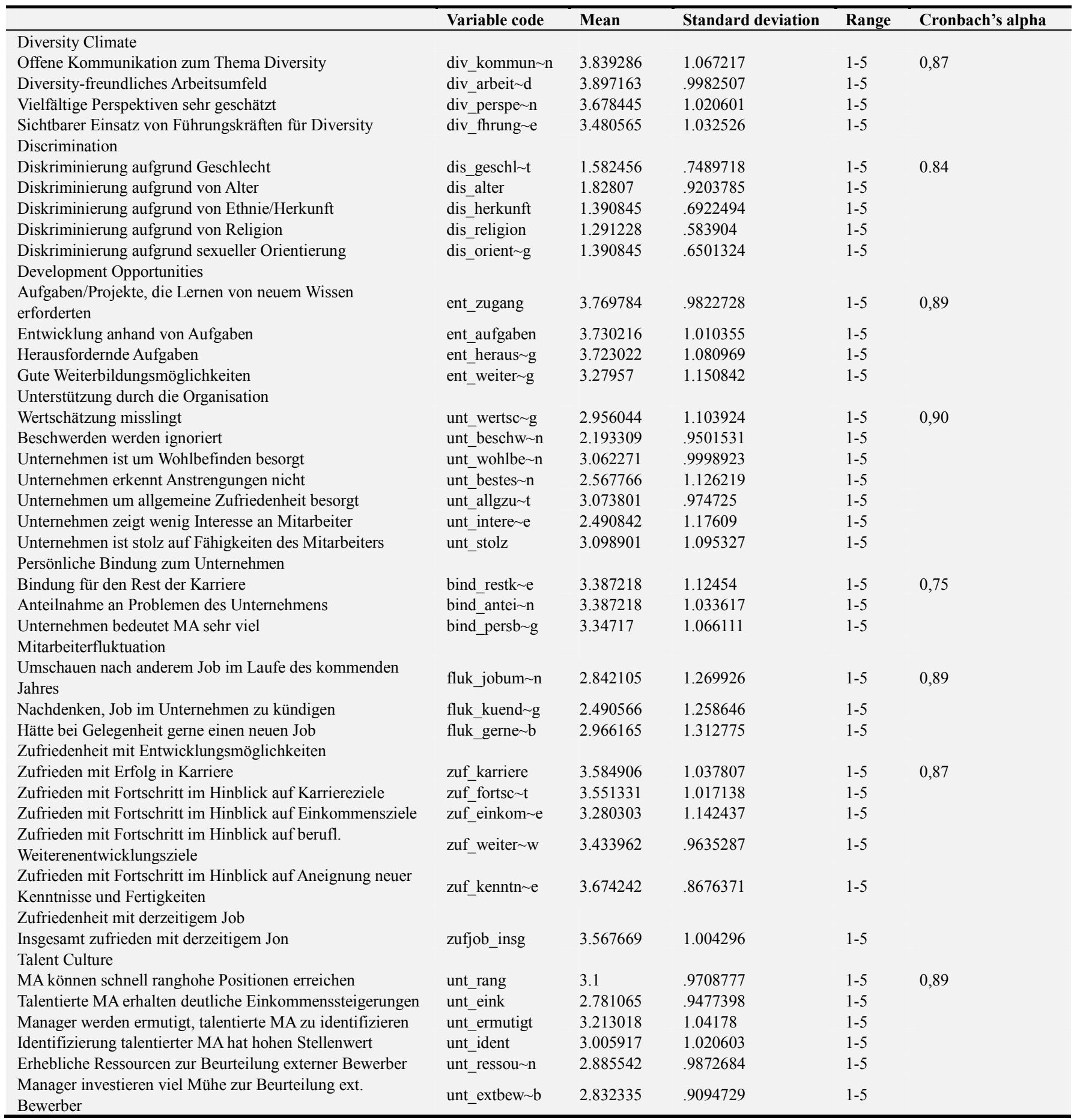




\begin{tabular}{|c|c|c|c|c|c|}
\hline & Variable code & Mean & Standard deviation & Range & Cronbach's alpha \\
\hline Talentierte MA erhalten herausfordernde Aufgaben & unt_heraus & 3.319527 & 9021982 & $1-5$ & \\
\hline Weiterbildungsmöglichkeiten exklusiv für talentierte MA & unt_weiter $\sim \mathrm{s}$ & 3.059524 & 1.103627 & $1-5$ & \\
\hline $\begin{array}{l}\text { Talentierte MA werden ermutigt, zwischen verschiedenen } \\
\text { Bereichen, Funktionen und Ländern zu wechseln }\end{array}$ & unt_wechsel & 3.094118 & 1.167849 & $1-5$ & \\
\hline $\begin{array}{l}\text { Verfahren, talentierte Angestellte im ganzen Unternehmen } \\
\text { sichtbar zu machen }\end{array}$ & unt_sichtbar & 2.751037 & 1.160212 & $1-5$ & \\
\hline
\end{tabular}

Table 1c provides an overview over the use of instruments from an employee and employer perspective. Interestingly, there are some variations due to different perceptions, partially due to the fact that not employees from all companies participated in the survey. Also, employees tend to rank instruments higher that they wish where offered in their organizations while they actually are not, whereas the once that are actually offered and not marked. So employees tend to rate what they wish rather than what is actually there. In the case of Age and Ethnic diversity, use of measures were assessed higher by employees in all cases.

Table 1c. Use of diversity instruments from employer and employee perspectives.

\begin{tabular}{|c|c|c|c|c|c|}
\hline & \multirow[b]{2}{*}{ Variable code } & \multicolumn{2}{|c|}{ Company Survey } & \multicolumn{2}{|c|}{ Employee Survey } \\
\hline & & Mean & Standard deviation & Mean & Standard deviation \\
\hline \multicolumn{6}{|l|}{ Gender Diversity } \\
\hline Part time contracts after parental leave & teilz_eltern & 4.215385 & .8748626 & 4.023622 & .7798304 \\
\hline Working from home & arb_zuhause & 3.296875 & 1.177564 & 3.23622 & 1.141781 \\
\hline Job sharing for leadership roles & Jobsharing & 1.676923 & .9032527 & 2.405622 & 1.055004 \\
\hline Networks for female leadership roles & netzwerke_ $\sim 1$ & 2.546875 & 1.390782 & 3.402439 & 1.217489 \\
\hline Mentoring & ment_weibl & 2.703125 & 1.387926 & 3.342975 & 1.20946 \\
\hline Personal coaching & coach_weibl & 2.78125 & 1.338902 & 3.102459 & 1.159038 \\
\hline Company kindergarten & Kindertages & 2.125 & 1.374369 & 2.797619 & 1.309905 \\
\hline Support for child care financing & unt_priv_k $\sim \mathrm{s}$ & 3.123077 & 1.525898 & 2.251029 & 1.08652 \\
\hline Company counsel for family topics & betreibl_a p p & 2.078125 & 1.645626 & 3.143443 & 1.12213 \\
\hline Conversations after return from parental leave & Rckkehrgesp & 2.390625 & 1.280094 & 3.549587 & .9288036 \\
\hline Targeted recruiting of women & rekrut_weibl & 4.061538 & .9822952 & n.a. & n.a. \\
\hline \multicolumn{6}{|l|}{ Age Diversity } \\
\hline Flexible working hours for mature staff & flex_model $\sim t$ & 3.031746 & 1.24393 & 3.42449 & 1.112251 \\
\hline Part time & Alterstz & 3.241935 & 1.478651 & 3.881148 & .9107345 \\
\hline Systematic development for mature staff & syst_perso $\sim t$ & 1.968254 & 1.031258 & 2.563559 & 1.064008 \\
\hline Horizontal moves for mature staff & horiz_aelt & 2.349206 & .9698475 & 2.652542 & .9925434 \\
\hline Targeted design of mixed age teams & altersgemi $\sim \mathrm{t}$ & 1.854839 & .9382015 & n.a. & n.a. \\
\hline Ergonomic design of working space & ergo_aplatz & 3.596774 & 1.165905 & 3.506224 & 1.012918 \\
\hline Design of content for mature staff & alters_ges $\sim 1$ & 2.222222 & 1.007143 & 2.504202 & 1.070305 \\
\hline Leisure/sportive activities & freiz_ange $\sim t$ & 3.492063 & 1.401301 & 3.786008 & 1.057973 \\
\hline Targeted external recruiting of mature staff & rekrut_ext & 1.580645 & .7798358 & 1.878788 & .8662916 \\
\hline Personal health counseling & pers_gesund & 2.634921 & 1.428981 & 2.978903 & 1.121618 \\
\hline Life phase oriented personnel development & Lebensphasen & 2.063492 & 1.17601 & n.a. & n.a. \\
\hline $\begin{array}{l}\text { Targeted recruiting of professionals and management with } \\
\text { migration background }\end{array}$ & rekrut_fac $\sim$ a & 2.145161 & 1.113947 & 2.553648 & 1.041342 \\
\hline Targeted recruiting of trainees and graduates & rekrut_abs $\sim$ a & 2.065574 & 1.046723 & 2.599138 & 1.088547 \\
\hline Targeted design of international teams & internat_t $\sim \mathrm{m}$ & 2.741935 & 1.317418 & n.a. & n.a. \\
\hline Job rotation/mobility programs & entsend_au $\sim$ d & 2.822581 & 1.261332 & 3.245763 & 1.136897 \\
\hline International experience requirement & auslandser $\sim \mathrm{s}$ & 2.409836 & 1.35864 & 2.798319 & 1.222709 \\
\hline Global talent pools & glob_talen $\sim$ s & 2.516129 & 1.501277 & 2.85654 & 1.170112 \\
\hline Trainings/Workshops with international focus & train_int_ $\sim \mathrm{S}$ & 2.774194 & 1.285727 & 2.752137 & 1.224962 \\
\hline Mentoring for international staff & ment_ausl & 2.290323 & 1.092254 & 2.582609 & 1.113495 \\
\hline Department to support international staff & abtl_unt_a a & 2.677419 & 1.544681 & 2.647826 & 1.215752 \\
\hline
\end{tabular}

Table 1d. provides a complete overview over the use of talent management instruments. 
Table 1d. Use of talent instruments.

\begin{tabular}{|c|c|c|c|c|}
\hline & Variable code & Mean & Standard deviation & Cronbach's Alpha \\
\hline \multicolumn{5}{|l|}{ External Recruiting } \\
\hline Detailed job requirements including competencies for new jobs & anfordprof $\sim p$ & 3.741935 & 1.085455 & \multirow[t]{12}{*}{0,65} \\
\hline Structured interviews & struk_interw & 4.129032 & .9318388 & \\
\hline Multiple interviews per applicant & mehrer_int $\sim v$ & 4.163934 & 9691977 & \\
\hline Written tests to measure cognitive skills & schriftl_kog & 2.590164 & 1.282927 & \\
\hline Written tests to measure professional knowledge & schriftl_f $\sim \mathrm{k}$ & 2.196721 & 1.166471 & \\
\hline Written tests to assess personality & schriftl_p $\sim$ s & 2.377049 & 1.331214 & \\
\hline External references & ext_ref & 2.633333 & .9382036 & \\
\hline Asesssment center & ass_cent & 3.096774 & 1.276646 & \\
\hline Active recruiting(head hunting) & Headhunting & 3.283333 & .9404591 & \\
\hline Targeted, external recruiting for talent pools/ succession plans & ext_rek_ta 1 & 2.66129 & 1.305016 & \\
\hline Refrer-a-friend programs & ref_friend & 2.516129 & 1.315007 & \\
\hline Targeted use of social media (Facebook, Xing, Linkedin etc.) & Socialmed & 2.677419 & 1.315409 & \\
\hline \multicolumn{5}{|l|}{ Internal Talent Identification } \\
\hline Structured assessment of potential by competency & struk_pote $\sim \mathrm{W}$ & 3.672131 & 1.220947 & \multirow[t]{8}{*}{0,67} \\
\hline Structured and regular talent review workshops/panels & syst_bespr $\sim \mathrm{h}$ & 3.655738 & 1.302373 & \\
\hline 360 degree feedback & Grad & 2.639344 & 1.252102 & \\
\hline Internal assessment center & int_ass_cent & 2.854839 & 1.35316 & \\
\hline Internal personal assessment & int_einzel $\sim \mathrm{s}$ & 2.52459 & 1.191729 & \\
\hline Personal assessment through external consultant & einzel_ass $\sim t$ & 2.213115 & 1.127161 & \\
\hline Talent pools for specialists and management roles & talent_poo $\sim r$ & 3.306452 & 1.26217 & \\
\hline Internal job portal & int_stelle $\mathrm{t}$ & 4.322581 & 1.004485 & \\
\hline \multicolumn{5}{|l|}{ Performance Management } \\
\hline Regular assessment based on quantitative scale & beurt_skala & 3.918033 & 1.429395 & \multirow[t]{6}{*}{0,62} \\
\hline Separate assessment of performance and potential & sepa_beurt 1 & 4 & 1.197219 & \\
\hline Target agreements & Zielvereinb & 4.516667 & .7700246 & \\
\hline Recommended distribution & empf_verte $\mathrm{g}$ & 2.65 & 1.470904 & \\
\hline Forced distribution & erzw_verte g & 1.766667 & 1.15519 & \\
\hline Performance panels with multiple leaders & beurt_konf $\sim \mathrm{n}$ & 3 & 1.527525 & \\
\hline \multicolumn{5}{|l|}{ Development } \\
\hline Personal development plans & pers_entwp $\sim$ e & 3.683333 & 9998587 & \multirow[t]{12}{*}{0,85} \\
\hline Mentoring & Mentoring & 3.033333 & .9560985 & \\
\hline Coaching & Coaching & 3.066667 & .7561425 & \\
\hline Job rotation & job_rotat & 2.666667 & 1.036073 & \\
\hline Stretch assignment as targeted development instrument & herausf_proj & 3.423729 & 1.101776 & \\
\hline Expat assignments & ausl_entsend & 2.6 & 1.044761 & \\
\hline Cross divisional career paths & bereichsub e & 2.672414 & 1.098451 & \\
\hline Assessment center/development center & ass_cent_e $\sim$ u & 3.05 & 1.43119 & \\
\hline Employee portal for training and development & mitportal_ b & 3.766667 & 1.382293 & \\
\hline Self assessment of employees & selbstbew_ d & 2.728814 & 1.243334 & \\
\hline Special portals for talent networks, communities, specialists & spez_portale & 1.847458 & .8672899 & \\
\hline Development programs for high potentials & entw_progr $\sim t$ & 3.45 & 1.28122 & \\
\hline \multicolumn{5}{|l|}{ Competency Model } \\
\hline Competency model & Kompmodell & .8245614 & .3837227 & \multirow[t]{7}{*}{0,79} \\
\hline Competency model is used for external selection & persauswah $\sim \mathrm{f}$ & 3.520833 & 1.091351 & \\
\hline Competency model is used for internal assignments & int_stellbe & 3.520833 & 1.091351 & \\
\hline Competency model is used for develop & entw_plan & 3.854167 & .9450787 & \\
\hline Continuous monitoring of development plans & pruef_entw $\sim$ e & 2.982759 & 1.177142 & \\
\hline Particularly talented employee participate in personal development & steuer_tal b & 3.474576 & 1.134961 & \\
\hline Personal development is evenly available to all employees & var3 & 3.20339 & 1.171201 & \\
\hline \multicolumn{5}{|l|}{ Retention } \\
\hline Flexible time models & flex_arbei 1 & 3.583333 & .9440566 & \multirow[t]{11}{*}{0,84} \\
\hline Sabbaticals & Sabbatic & 2.237288 & .9161197 & \\
\hline Professional studies & berufsbegl $\sim d$ & 2.916667 & 1.013329 & \\
\hline Special compensation for talents & spez_verguet & 1.813559 & 1.008149 & \\
\hline Individual career planning & indiv_karr $\sim \mathrm{n}$ & 3.216667 & 1.075011 & \\
\hline Regular employee surveys for talents & talentpool $\sim \mathrm{g}$ & 2.016667 & 1.200165 & \\
\hline Assessment of intention to leave & Abwandrisik & 2.183333 & 1.016669 & \\
\hline Personalized career paths & perso_lauf $\sim \mathrm{f}$ & 2.338983 & 1.183364 & \\
\hline Alternative career paths & alter_karr $\sim$ f & 2.9 & 1.258193 & \\
\hline Structured exit management & exit_manag & 2.433333 & 1.357548 & \\
\hline Structured conversation after leave of absence & strukt_rue $\sim \mathrm{r}$ & 2.448276 & 1.40391 & \\
\hline
\end{tabular}

Table $2 \mathrm{a}$ and $\mathrm{b}$ show diversity metrics both from an employer and employee perspectives, contrasting the results of the employer and employee survey. Table $2 \mathrm{a}$ and $\mathrm{b}$ show some of the diversity metrics. 
Table 2a. Talent and diversity metrics (employer perspective).

\begin{tabular}{|c|c|c|c|c|}
\hline & Variable code & Mean & Standard deviation & Cronbach's Alpha \\
\hline \multicolumn{5}{|l|}{ Gender diversity metrics } \\
\hline Gender quota (all employees) & frauenquot $\sim \mathrm{t}$ & 32 & 16.62471 & n.a. \\
\hline Gender quota (leadership) & frauenquot 3 & 11.14634 & 8.987661 & \\
\hline Gender quota (non union employees) & frauenquot $\mathrm{f}$ & 17.94737 & 11.65715 & \\
\hline Gender quota (new hires) & anteil_fra $\sim 1$ & 32.37143 & 17.37321 & \\
\hline Gender quota part time workers & ant_frauen $\sim$ Z & 30.42424 & 17.76589 & \\
\hline Percent of male parents taking leave of absence & maenl_ellt $\sim t$ & 13.83871 & 18.43746 & \\
\hline Training days & weiterbild e & 4.814815 & 2.774015 & \\
\hline \multicolumn{5}{|l|}{ Age diversity metrics } \\
\hline Percentage age $15-25$ & ant 1525 & 8.967742 & 4.607847 & n.a. \\
\hline Percentage age $26-35$ & ant26_35 & 22.22581 & 11.44468 & \\
\hline Percentage age $36-45$ & ant36_45 & 31.46667 & 8.386086 & \\
\hline Percentage age $46-55$ & ant46_55 & 26.77419 & 10.69489 & \\
\hline Percentage age 56-66 & ant56_65 & 11.35484 & 5.320078 & \\
\hline Quota international staff & ant_nichtd $\sim \mathrm{t}$ & 20.93939 & 17.59783 & n.a. \\
\hline \multicolumn{5}{|l|}{ Other metrics } \\
\hline Duration of parental leave in years & dauer_elte 1 & 1.925926 & 2.758566 & n.a. \\
\hline Duration of parental leave on months & Monate & 5.857143 & 4.00357 & \\
\hline External recruiting rate top and senior management & extbes_top | & 20.32143 & 17.03168 & \\
\hline External recruiting rate of middle managers & extbes_mm | & 20 & 9.873271 & \\
\hline External recruiting rate other non union employees & extbes_and $\sim t$ & 30.7037 & 17.12132 & \\
\hline External recruiting rate specialists & $\operatorname{var} 5$ & 30.07143 & 17.09869 & \\
\hline
\end{tabular}

Table 2b. Talent and diversity metrics (employee perspective).

\begin{tabular}{|c|c|c|c|c|}
\hline & Variable code & Mean & Standard deviation & Cronbach's Alpha \\
\hline Are you a member of a talent pool (to the best of your knowledge) & talentpool d & 1.733607 & .442981 & n.a. \\
\hline How many years in current position & beantwortu $\sim \mathrm{d}$ & 5.813953 & 5.058024 & \\
\hline Time elapsed since last promotion & $\operatorname{var} 12$ & 16.95322 & 10.1887 & \\
\hline Time expected until next promotion & var22 & 7.458333 & 5.797846 & \\
\hline Time desired until next promotion & $\operatorname{Var} 32$ & 8.39521 & 4.020966 & \\
\hline I am an outgoing and social person & ich_heraus $\sim \mathrm{n}$ & 3.917355 & .8156842 & \\
\hline I am someone who works diligently & ich_grndlich & 4.115226 & .694648 & \\
\hline I am someone who effectively and efficiently & ich_wirksam & 4.368852 & .6311635 & \\
\hline I am a somewhat lazy person & ich_faul & 1.632231 & .7519507 & \\
\hline I am easily irritated & ich_nervoes & 2.065844 & .8694695 & \\
\hline I am someone who worries easily & ich_sorgen & 2.471074 & .9473648 & \\
\hline I am relaxed and can deal with stress easily & ich_entspa $\mathrm{t}$ & 3.646091 & .8943435 & \\
\hline I am respectful and friendly with others & ich_freund $\sim \mathrm{h}$ & 4.278689 & .739951 & \\
\hline I am sometimes a bit rough with others & ich_grob & 2.385892 & .9897644 & \\
\hline I have a lively phantasy and imagination & ich_phanta e & 3.5 & 1.024394 & \\
\hline I am an original character & ich_origin $\sim 1$ & 3.8107 & .8113151 & \\
\hline I value artistic experiences & ich_kuenst $\sim$ e & 3.07438 & 1.067555 & \\
\hline Sex & geschlecht & 1.367769 & .4831973 & \\
\hline Age & alter & 37.39916 & 9.335536 & \\
\hline Education level & bildung & 2.805785 & .5148093 & \\
\hline Employer & arbeitgeber & n.a. & n.a. & \\
\hline Management responsibility & fuehrungsv $\sim \mathrm{g}$ & 1.643154 & .480066 & \\
\hline Responsible for how many people & unterstell a & 18.49333 & 28.82256 & \\
\hline
\end{tabular}

In order to test the hypothesis about the effect of the use of certain instruments on certain diversity measures, regression analyses were carried out with different dependent variables: table 3 gives an overview over means, standard deviations and correlations for all variables. For gender diversity, table 3 a shows the effect on the general gender quota and the leadership quota. Table $3 \mathrm{~b}$ shows the effect of age and ethnic diversity instruments on age distribution and ethnic diversity. 
Table 3. Means, standard deviations and correlations for all variables.

\begin{tabular}{|c|c|c|c|c|c|c|c|c|c|}
\hline Variables & Mean & Standard deviation & gender & age & ethnis & frauenquot $\sim t$ & frauenquot $\sim 3$ & frauenquot $\mathbf{f}$ & anteil_fra $\sim 1$ \\
\hline gender & 2.988095 & .670636 & 1.0000 & & & & & & \\
\hline age & 2.492424 & .5663469 & 0.2422 & 1.0000 & & & & & \\
\hline ethnis & 2.775 & .8080561 & 0.0857 & 0.0673 & 1.0000 & & & & \\
\hline frauenquot $\sim t$ & 35.08333 & 21.16798 & -0.2350 & -0.2016 & -0.5882 & 1.0000 & & & \\
\hline frauenquot $\sim 3$ & 11.75 & 11.63166 & -0.0096 & 0.0806 & -0.3712 & 0.3896 & 1.0000 & & \\
\hline frauenquot $\sim \mathrm{f}$ & 20.08333 & 14.56932 & -0.0630 & -0.1056 & -0.4871 & 0.6594 & 0.8810 & 1.0000 & \\
\hline anteil_fra 1 & 38.25 & 21.42269 & -0.0016 & -0.2147 & -0.5652 & 0.8882 & 0.4103 & 0.6617 & 1.0000 \\
\hline ant_frauen $\sim Z$ & 27.58333 & 11.828 & -0.3355 & 0.1679 & 0.1234 & 0.0408 & -0.5136 & -0.2124 & -0.0473 \\
\hline ant_nichtd $\sim \mathrm{t}$ & 20.75 & 18.3755 & -0.4492 & -0.1396 & 0.0044 & 0.2763 & -0.0394 & 0.0728 & 0.3378 \\
\hline maenl_ellt $\sim t$ & 10.25 & 16.68491 & 0.1999 & 0.1747 & -0.2416 & -0.1326 & 0.1620 & -0.0629 & -0.1576 \\
\hline aussertari $\sim 1$ & 17.91667 & 14.53184 & 0.3737 & 0.3971 & 0.0029 & -0.0511 & 0.0881 & -0.0850 & 0.0106 \\
\hline weiterbild $\sim$ e & 3.916667 & 2.065224 & 0.2243 & 0.0312 & -0.1484 & -0.1392 & 0.0558 & 0.0577 & -0.2789 \\
\hline dauer_elte 1 & 1.583333 & 1.1645 & -0.0818 & -0.0741 & 0.2005 & -0.1681 & -0.5655 & -0.3139 & -0.1922 \\
\hline ant_ausser $\sim a$ & 24.75 & 31.83801 & -0.3797 & -0.7580 & 0.0697 & 0.1063 & -0.3610 & -0.1275 & -0.0386 \\
\hline ma_ausl & 71965.83 & 83570.2 & 0.1623 & -0.5120 & 0.3004 & 0.0351 & -0.3615 & -0.0600 & 0.1467 \\
\hline fehltage_k k k & 7.5 & 4.871065 & 0.5427 & 0.3490 & 0.0543 & -0.3134 & -0.1420 & -0.2376 & -0.2365 \\
\hline ma_fluk & 7.833333 & 8.515583 & -0.5109 & -0.4321 & -0.3362 & 0.6295 & 0.0675 & 0.3540 & 0.6590 \\
\hline
\end{tabular}

Table 3. Continue.

\begin{tabular}{|c|c|c|c|c|c|c|c|c|c|c|}
\hline Variables & ant_frauen $\sim \mathbf{Z}$ & ant_nichtd $\sim t$ & maenl_ellt $\sim t$ & aussertari $\sim$ & weiterbild $\sim$ e & dauer_elte I & ant_ausser a & ma_ausl & fehltage_k $\sim k$ & ma_fluk \\
\hline \multicolumn{11}{|l|}{ gender } \\
\hline \multicolumn{11}{|l|}{ age } \\
\hline \multicolumn{11}{|l|}{ ethnis } \\
\hline \multicolumn{11}{|l|}{ frauenquot $\sim t$} \\
\hline \multicolumn{11}{|l|}{ frauenquot $\sim 3$} \\
\hline \multicolumn{11}{|l|}{ frauenquot $\sim \mathrm{f}$} \\
\hline \multicolumn{11}{|l|}{ anteil_fra 1 } \\
\hline ant_frauen $\sim \mathrm{Z}$ & 1.0000 & & & & & & & & & \\
\hline ant_nichtd $\sim t$ & 0.2437 & 1.0000 & & & & & & & & \\
\hline maenl_ellt $\sim t$ & -0.3638 & -0.1990 & 1.0000 & & & & & & & \\
\hline aussertari 1 & -0.1748 & -0.6132 & 0.3960 & 1.0000 & & & & & & \\
\hline weiterbild $\sim$ e & 0.0357 & -0.5420 & 0.6708 & 0.3269 & 1.0000 & & & & & \\
\hline dauer_elte 1 & 0.6925 & -0.2942 & -0.3731 & -0.0022 & 0.1355 & 1.0000 & & & & \\
\hline ant_ausser $\sim a$ & 0.2539 & -0.0192 & -0.1857 & -0.1663 & 0.2209 & 0.3157 & 1.0000 & & & \\
\hline ma_ausl & 0.3710 & 0.2836 & -0.3369 & -0.4002 & -0.0259 & 0.3523 & 0.4019 & 1.0000 & & \\
\hline fehltage_k $\sim \mathrm{k}$ & 0.0434 & -0.2879 & 0.6057 & 0.3564 & 0.5558 & 0.0881 & -0.3555 & 0.2015 & 1.0000 & \\
\hline ma_fluk & 0.2411 & 0.6754 & -0.2473 & -0.4218 & -0.3369 & -0.0076 & 0.3335 & 0.1649 & -0.5896 & 1.0000 \\
\hline
\end{tabular}

Table 3a. Regression analysis (employer survey): effect of gender instruments on overall female quota (model 1) and female leadership positions (model b).

\begin{tabular}{|c|c|c|c|c|c|}
\hline & \multirow[t]{2}{*}{ Variable code } & & \multirow{2}{*}{$\begin{array}{c}\text { Regression model a } \\
\mathbf{p}\end{array}$} & \multicolumn{2}{|c|}{ Regression model b } \\
\hline & & Beta & & Beta & $\mathbf{p}$ \\
\hline Flexible working hours & flex_arbei $\sim \mathrm{V}$ & 16.68044 & 0.014 & 2.402103 & 0.608 \\
\hline Part time contracts after parental leave & teilz_elte $\sim \mathrm{V}$ & 3.157783 & 0.660 & 3.090538 & 0.525 \\
\hline Working from home & arb_zuhaus $\sim \mathrm{V}$ & .9479923 & 0.893 & -8.108065 & 0.073 \\
\hline Job sharing for leadership roles & jobsharing $\sim \mathrm{V}$ & -2.172129 & 0.887 & 3.083643 & 0.828 \\
\hline Diversity trainings & train_dive $\sim \mathrm{V}$ & -3.725093 & 0.764 & -7.098527 & 0.398 \\
\hline Networks for female leadership roles & netzwerke_ $\sim \mathrm{V}$ & 7.001714 & 0.562 & -8.081704 & 0.306 \\
\hline Mentoring & ment_weibl $\sim \mathrm{V}$ & -4.979274 & 0.594 & -4.589263 & 0.449 \\
\hline Personal coaching & coach_weib $\sim v$ & 1.67306 & 0.550 & 7.972963 & 0.128 \\
\hline Company kindergarten & kindertage $\sim \mathrm{v}$ & 2.940117 & 0.760 & -2.763412 & 0.656 \\
\hline Support for child care financing & unt_priv_k $\sim \mathrm{V}$ & -2.038495 & 0.857 & -2.28303 & 0.777 \\
\hline Company counsel for family topics & betreibl_a $\sim \mathrm{v}$ & .2565389 & 0.976 & -3.520359 & 0.532 \\
\hline Quota for female managers & quote_weib $\sim v$ & -2.922899 & 0.699 & -.105131 & 0.983 \\
\hline Conversations after return from parental leave & rckkehrges $\sim \mathrm{V}$ & 1.089929 & 0.857 & 7.497415 & 0.072 \\
\hline Targeted recruiting of women & rekrut_wei $\sim$ V & -3.280317 & 0.720 & 4.498514 & 0.448 \\
\hline
\end{tabular}


Table 3b. Regression analysis: Model a: Effect of age diversity instruments on percentage of employees 56 - 65 years Model b: Effect of ethnic diversity instruments on percentage of non-German employees.

\begin{tabular}{|c|c|c|c|c|c|}
\hline & \multirow[t]{2}{*}{ Variable code } & \multicolumn{2}{|c|}{ Regression model a } & \multicolumn{2}{|c|}{ Regression model b } \\
\hline & & Beta & $\mathbf{p}$ & Beta & $\mathbf{p}$ \\
\hline Flexible working hours for mature staff & flex_model $\sim v$ & .0170212 & 0.725 & & \\
\hline Part time for mature staff & alterstz_av & .0393844 & 0.456 & & \\
\hline Systematic development for mature staff & syst_perso v & -.2283264 & 0.146 & & \\
\hline Horizontal moves for mature staff & horiz aelt $\sim \mathrm{V}$ & .1768819 & 0.061 & & \\
\hline Targeted design of mixed age teams & altersgemi $\sim \mathrm{V}$ & .0181552 & 0.916 & & \\
\hline Ergonomic design of working space & ergo_aplat $\sim v$ & .0457892 & 0.674 & & \\
\hline Design of content for mature staff & alters_ges $\sim v$ & .0579999 & 0.561 & & \\
\hline Leisure/sports activities & freiz_ange $\sim v$ & .0351066 & 0.718 & & \\
\hline Targeted external recruiting of mature staff & rekrut_ext $\sim \mathrm{V}$ & n.a. & n.a. & & \\
\hline Personal health counseling & pers_gesun $\sim \mathrm{V}$ & -.0287659 & 0.725 & & \\
\hline Life phase oriented personnel development & lebensphas $\sim v$ & .0103078 & 0.936 & & \\
\hline Targeted recruiting of foreign professionals & rekrut_aus $\sim \mathrm{V}$ & & & .3497993 & 0.031 \\
\hline Targeted recruiting of professionals and management with migration background & rekrut_fac $\sim v$ & & & .3913477 & 0.066 \\
\hline Targeted recruiting of trainees and graduates & rekrut_abs $\sim v$ & & & .4176494 & 0.106 \\
\hline Targeted design of international teams & internat $t \sim v$ & & & .4277092 & 0.013 \\
\hline Job rotation / mobility programs & entsend_au $\sim v$ & & & -.2163998 & 0.333 \\
\hline \multirow[t]{2}{*}{ International experience requirement } & auslandser $\sim \mathrm{v}$ & & & .3347404 & 0.126 \\
\hline & abovemed_eth & & & .2145455 & 0.213 \\
\hline Global talent pools & glob_talen $\sim v$ & & & .0704411 & 0.711 \\
\hline Trainings / Workshops with international focus & train_int_ $\sim \mathrm{V}$ & & & .1479849 & 0.450 \\
\hline Mentoring for international staff & ment_ausl_av & & & -.0682234 & 0.740 \\
\hline Department to support international staff & abtl_unt $\mathrm{a} \sim \mathrm{v}$ & & & -.1216473 & 0.607 \\
\hline
\end{tabular}

Table 4 shows the results of a regression for the use of specific instruments on various aspects of diversity culture from an employee perspective, such as perception on diversity (model a), career and development opportunities (model b), sense of embeddedness and recognition (model c), personal commitment and contentedness (model d) and finally perception on work life balance (model e). Results show that surprisingly, the use of diversity measures is overall most positively connected with a positive perception on the career and development opportunities in the organization Table 5 mirrors the results from an employer perspective.

Table 4. Results of regression analysis (employee survey): effects of instrument use on the perception of the diversity climate (model a), career and development opportunities (model b), sense of embeddedness and recognition (model c), personal commitment (model d), and perception of work life balance (model e).

\begin{tabular}{|c|c|c|c|c|c|}
\hline & \multirow[b]{2}{*}{ Variable code } & \multicolumn{2}{|c|}{ Model a Variable: Perception } & \multicolumn{2}{|c|}{ Model b Variable: Career opport. } \\
\hline & & Beta & $\mathbf{P}$ & Beta & $\mathbf{P}$ \\
\hline Flexible working hours & arbeitszei $\sim \mathrm{n}$ & .1021195 & $0.006 * * *$ & .2767632 & $0.002 * * *$ \\
\hline Part time contracts after parental leave & teilzeitve $\sim \mathrm{e}$ & -.0332391 & 0.479 & -.113108 & 0.311 \\
\hline Working from home & arbeitenzu e & -.0886579 & $0.016 * *$ & -.1274645 & 0.141 \\
\hline Job sharing for leadership roles & jobsharing & .0837908 & $0.024 * *$ & .1118814 & 0.199 \\
\hline Diversity trainings & trainingdi $\sim \mathrm{g}$ & .0795853 & $0.031 * *$ & .157603 & $0.070 *$ \\
\hline Networks for female leadership roles & netzwerke & .0058554 & 0.922 & -.1275864 & 0.367 \\
\hline Personal coaching & coaching & -.0114434 & 0.811 & -.0487254 & 0.668 \\
\hline Company kindergarten & kindertage $\sim \mathrm{n}$ & .004784 & 0.869 & -.046608 & 0.497 \\
\hline Support for child care financing & kinderbetr $\sim \mathrm{n}$ & -.0493814 & 0.179 & -.1417097 & 0.105 \\
\hline Company counsel for family topics & ansprechpa $\sim \mathrm{r}$ & .0694223 & $0.020 * *$ & .1754703 & $0.014 * *$ \\
\hline Quota for female managers & quote & .0147556 & 0.577 & .0506689 & 0.420 \\
\hline Conversations after return from parental leave & rckkehrges $\sim$ e & .0400626 & 0.355 & .1005941 & 0.328 \\
\hline Flexible working hours for mature staff & alt_arbei le & .0330905 & 0.404 & .0649683 & 0.489 \\
\hline Part time for mature staff & alt_alter it & -.0519042 & 0.227 & -.0660551 & 0.516 \\
\hline Horizontal moves for mature staff & alt_horizo $\sim 1$ & .0321177 & 0.522 & .0248237 & 0.835 \\
\hline Targeted design of mixed age teams & alt_alter $\sim$ ht & .0698129 & 0.121 & .1217546 & 0.254 \\
\hline Ergonomic design of working space & alt_ergo & -.0391231 & 0.295 & -.030985 & 0.726 \\
\hline Design of content for mature staff & alt_arbei te & -.1319107 & $0.005 * * *$ & -.3087533 & $0.006 * * *$ \\
\hline Leisure/sports activities & alt_freize $\sim$ e & .0348108 & 0.374 & .0965923 & 0.299 \\
\hline Targeted external recruiting of mature staff & alt_rekrut $\sim g$ & -.045675 & 0.318 & -.1956679 & $0.073 *$ \\
\hline Personal health counseling & alt_gesund $\sim g$ & -.0140041 & 0.692 & .0300406 & 0.721 \\
\hline Targeted recruiting of foreign professionals & eth_ausl & .0764665 & 0.124 & .1665534 & 0.158 \\
\hline $\begin{array}{l}\text { Targeted recruiting of professionals and } \\
\text { management with migration background }\end{array}$ & eth_migra & -.0274161 & 0.752 & -.1375271 & 0.505 \\
\hline Targeted recruiting of trainees and graduates & eth_azubi & -.0639619 & 0.395 & -.0497536 & 0.780 \\
\hline
\end{tabular}




\begin{tabular}{llllll}
\hline & & \multicolumn{2}{l}{ Model a Variable: Perception } & \multicolumn{2}{c}{ Model b Variable: Career opport. } \\
\hline & Variable code & Beta & P & Beta & \multicolumn{2}{l}{ P } \\
\hline Job rotation / mobility programs & eth_rotation & .0722863 & $0.076^{* *}$ & .1499326 & 0.121 \\
International experience requirement & eth_voraus & -.0279655 & 0.456 & -.0331452 & 0.710 \\
Global talent pools & eth_globale & .0418629 & 0.335 & .061676 & 0.549 \\
Trainings / Workshops with international focus & eth_training & .0924539 & $0.023^{* *}$ & .1246749 & 0.193 \\
Mentoring for international staff & eth_mentor g & -.1441717 & $0.002^{* * *}$ & -.1053757 & 0.337 \\
Department to support foreign professionals & eth_abteil g & .027437 & 0.427 & .0513389 & 0.531 \\
& cons & 1.865497 & 0.000 & 1.193816 & 0.016 \\
\hline
\end{tabular}

Table 4. Continue.

\begin{tabular}{|c|c|c|c|c|c|c|}
\hline & \multicolumn{2}{|c|}{$\begin{array}{l}\text { Model c Variable: } \\
\text { Embeddedness }\end{array}$} & \multicolumn{2}{|c|}{$\begin{array}{l}\text { Model d Variable: } \\
\text { Commitment }\end{array}$} & \multicolumn{2}{|c|}{$\begin{array}{l}\text { Model d Variable: Work } \\
\text { life balance }\end{array}$} \\
\hline & Beta & $\mathbf{P}$ & Beta & $\mathbf{P}$ & Beta & $\mathbf{P}$ \\
\hline Flexible working hours & .0033908 & 0.944 & .0927174 & $0.020 * *$ & -.0720385 & 0.359 \\
\hline Part time contracts after parental leave & .0231959 & 0.710 & -.0165088 & 0.744 & -.2858656 & $0.005 * * *$ \\
\hline Working from home & .0113375 & 0.816 & .0471598 & 0.231 & .091162 & 0.242 \\
\hline Job sharing for leadership roles & -.0448663 & 0.354 & -.0173663 & 0.654 & .0256412 & 0.738 \\
\hline Diversity trainings & -.0988767 & $0.039 * *$ & .052534 & 0.172 & -.0552258 & 0.469 \\
\hline Networks for female leadership roles & .0833449 & 0.295 & -.0601219 & 0.348 & .1734503 & 0.173 \\
\hline Mentoring & -.0958857 & 0.241 & .0911911 & 0.168 & -.0343112 & 0.792 \\
\hline Personal coaching & -.0032057 & 0.960 & -.0637581 & 0.215 & .0938503 & 0.356 \\
\hline Company kindergarten & -.0187318 & 0.635 & -.0722233 & 0.022 & -.0225347 & 0.716 \\
\hline Support for child care financing & -.0102658 & 0.834 & -.0502528 & 0.203 & .0018441 & 0.981 \\
\hline Company counsel for family topics & .047244 & 0.230 & .0450899 & 0.159 & -.0513483 & 0.420 \\
\hline Quota for female managers & .0674056 & $0.056^{* *}$ & .0526323 & 0.064 & .0459627 & 0.416 \\
\hline Conversations after return from parental leave & -.0223023 & 0.699 & -.0026874 & 0.954 & -.001683 & 0.986 \\
\hline Flexible working hours for mature staff & -.022751 & 0.665 & -.0279965 & 0.510 & .0798747 & 0.346 \\
\hline Part time for mature staff & .0163062 & 0.775 & .0536813 & 0.246 & .012745 & 0.889 \\
\hline Systematic development for mature staff & -.0479899 & 0.446 & .013637 & 0.789 & .0767834 & 0.447 \\
\hline Horizontal moves for mature staff & .0978127 & 0.147 & .0479924 & 0.376 & -.1244838 & 0.248 \\
\hline Targeted design of mixed age teams & .0002051 & 0.997 & .0475695 & 0.326 & .064805 & 0.499 \\
\hline Ergonomic design of working space & -.0033 & 0.948 & .0045427 & 0.910 & .0872408 & 0.278 \\
\hline Design of content for mature staff & -.0182261 & 0.779 & -.0227289 & 0.648 & -.0956129 & 0.333 \\
\hline Leisure/sports activities & -.1569358 & $0.004 * * *$ & .021989 & 0.602 & -.1638714 & $0.052 *$ \\
\hline Targeted external recruiting of mature staff & -.0689243 & 0.276 & -.0788937 & 0.108 & .0242173 & 0.803 \\
\hline Personal health counseling & .0691075 & 0.155 & -.0046309 & 0.903 & -.0183705 & 0.809 \\
\hline Targeted recruiting of foreign professionals & -.0574508 & 0.395 & -.1049836 & $0.050^{*}$ & .2981917 & $0.005 * * *$ \\
\hline $\begin{array}{l}\text { Targeted recruiting of professionals and management with } \\
\text { migration background }\end{array}$ & .0565319 & 0.624 & -.0054339 & 0.954 & -.5354586 & $0.005 * * *$ \\
\hline Targeted recruiting of trainees and graduates & .0218874 & 0.827 & .1029292 & 0.205 & .0944577 & 0.556 \\
\hline Job rotation / mobility programs & -.0209088 & 0.704 & .085002 & $0.054 *$ & .0205573 & 0.812 \\
\hline International experience requirement & -.0061805 & 0.902 & -.0775433 & $0.054^{*}$ & -.0328208 & 0.678 \\
\hline Global talent pools & -.0284482 & 0.615 & .0012402 & 0.978 & .1559565 & $0.087 * *$ \\
\hline Trainings / Workshops with international focus & -.0361386 & 0.492 & .0327643 & 0.440 & -.2380662 & $0.005 * * *$ \\
\hline Mentoring for international staff & .102689 & 0.107 & .0130416 & 0.790 & -.061034 & 0.530 \\
\hline Department to support foreign professionals & -.0723933 & 0.113 & -.0075143 & 0.836 & .0718104 & 0.318 \\
\hline & 3.992103 & 0.000 & 2.509912 & 0.000 & 3.60332 & 0.000 \\
\hline
\end{tabular}

Table 5. Results of regression analysis (company survey): effects of instrument use on the perception of the diversity climate (model a), career and development opportunities (model b), sense of embeddedness and recognition (model c), personal commitment (model d), and perception of work life balance (model e).

\begin{tabular}{|c|c|c|c|c|c|}
\hline & \multicolumn{3}{|c|}{$\begin{array}{l}\text { Model a Variable: } \\
\text { Perception [var 344] }\end{array}$} & \multicolumn{2}{|c|}{$\begin{array}{l}\text { Model b Variable: Career } \\
\text { opport. [var 345] }\end{array}$} \\
\hline & Variable code & Beta & $\mathbf{P}$ & Beta & $\mathbf{P}$ \\
\hline Flexible working hours & flex_arbei n & .0019203 & 0.914 & .002277 & 0.936 \\
\hline Part time contracts after parental leave & teilz_eltern & -.000452 & 0.986 & -.0032143 & 0.938 \\
\hline Working from home & arb_zuhause & -.0104615 & 0.528 & .0100187 & 0.704 \\
\hline Job sharing for leadership roles & jobsharing & .0393241 & $0.062 * *$ & .0639668 & $0.058 * *$ \\
\hline Diversity trainings & train_divers & -.0076768 & 0.698 & -.0060116 & 0.849 \\
\hline Networks for female leadership roles & netzwerke_ 1 & .0033994 & 0.831 & .0153596 & 0.549 \\
\hline Mentoring & ment_weibl & .0096361 & 0.589 & .0094947 & 0.738 \\
\hline Personal coaching & coach_weibl & .0081309 & 0.586 & .0119296 & 0.617 \\
\hline Company kindergarten & kindertages & -.0015497 & 0.924 & -.0110024 & 0.672 \\
\hline Support for child care financing & unt_priv_k $\sim \mathrm{s}$ & .0124761 & 0.289 & .0129382 & 0.486 \\
\hline Company counsel for family topics & betreibl_a $\sim p$ & -.0265828 & 0.123 & -.0509399 & $0.069 * *$ \\
\hline Quota for female managers & quote_weibl & .0034466 & 0.789 & .0239 & 0.255 \\
\hline
\end{tabular}




\begin{tabular}{|c|c|c|c|c|c|}
\hline & \multirow[b]{2}{*}{ Variable code } & \multirow[b]{2}{*}{ Beta } & \multirow{2}{*}{$\begin{array}{l}\begin{array}{l}\text { Model a Variable: } \\
\text { Perception [var 344] }\end{array} \\
\text { P }\end{array}$} & \multicolumn{2}{|c|}{$\begin{array}{l}\text { Model b Variable: Career } \\
\text { opport. [var 345] }\end{array}$} \\
\hline & & & & Beta & $\mathbf{P}$ \\
\hline Conversations after return from parental leave & rckkehrgesp & -.002393 & 0.869 & .0033942 & 0.884 \\
\hline Targeted recruiting of women & rekrut_weibl & -.0064437 & 0.719 & -.0209489 & 0.467 \\
\hline Flexible working hours for mature staff & flex_model $\sim \mathrm{t}$ & .0100147 & 0.603 & .034336 & 0.271 \\
\hline Part time for mature staff & alterstz & -.0096306 & 0.445 & -.0432366 & $0.044 * *$ \\
\hline Systematic development for mature staff & syst perso $\sim t$ & -.0269237 & 0.417 & -.014836 & 0.777 \\
\hline Horizontal moves for mature staff & horiz_aelt & -.0147236 & 0.528 & -.014809 & 0.690 \\
\hline Targeted design of mixed age teams & altersgemi $\sim \mathrm{t}$ & -.0239999 & 0.422 & -.089367 & $0.074 * *$ \\
\hline Ergonomic design of working space & ergo_aplatz & -.0069385 & 0.660 & -.0173353 & 0.493 \\
\hline Design of content for mature staff & alters_ges $\sim 1$ & -.0073482 & 0.773 & -.0029116 & 0.943 \\
\hline Leisure/sports activities & freiz_ange $\mathrm{t}$ & .0312377 & $0.045^{* *}$ & .048333 & $0.051^{* *}$ \\
\hline Targeted external recruiting of mature staff & rekrut_ext & -.0295333 & 0.284 & -.053682 & 0.226 \\
\hline Personal health counseling & pers_gesund & -.0042339 & 0.777 & .0105762 & 0.658 \\
\hline Life phase oriented personnel development & lebensphasen & .0157851 & 0.604 & .0090111 & 0.853 \\
\hline Targeted recruiting of foreign professionals & rekrut_ausl & .0052608 & 0.851 & .0556517 & 0.226 \\
\hline $\begin{array}{l}\text { Targeted recruiting of professionals and management with } \\
\text { migration background }\end{array}$ & rekrut_fac $\sim$ a & .0239445 & 0.600 & -.0250606 & 0.730 \\
\hline Targeted recruiting of trainees and graduates & rekrut_abs $\sim$ a & -.0005401 & 0.989 & .0584253 & 0.340 \\
\hline Targeted design of international teams & internat_t $\sim \mathrm{m}$ & -.0103669 & 0.648 & -.0729311 & $0.058 * *$ \\
\hline Job rotation / mobility programs & entsend_au $\sim$ d & -.0143934 & 0.443 & -.0289871 & 0.336 \\
\hline International experience requirement & auslandser $\sim \mathrm{s}$ & -.0036471 & 0.786 & -.0023635 & 0.912 \\
\hline Global talent pools & glob_talen $\sim$ s & -.0072367 & 0.652 & .00042 & 0.987 \\
\hline Trainings / Workshops with international focus & train_int_ $\sim \mathrm{s}$ & -.0103566 & 0.583 & -.0070068 & 0.815 \\
\hline Mentoring for international staff & ment_ausl & .0379866 & 0.218 & .0619392 & 0.209 \\
\hline \multirow[t]{2}{*}{ Department to support foreign professionals } & abtl_unt_a a a & .0047588 & 0.714 & .0038922 & 0.851 \\
\hline & cons & 2.963182 & 0.000 & 3.819733 & 0.000 \\
\hline
\end{tabular}

Table 5. Continue.

\begin{tabular}{|c|c|c|c|c|c|c|}
\hline & \multicolumn{2}{|c|}{$\begin{array}{l}\text { Model c Variable: } \\
\text { Embeddedness [var 346] }\end{array}$} & \multicolumn{2}{|c|}{$\begin{array}{l}\text { Model d Variable: } \\
\text { Commitment [var 347] }\end{array}$} & \multicolumn{2}{|c|}{$\begin{array}{l}\text { Model d Variable: Work life } \\
\text { balance [var 348] }\end{array}$} \\
\hline & Beta & $\mathbf{P}$ & Beta & $\mathbf{P}$ & Beta & $\mathbf{P}$ \\
\hline Flexible working hours & -.000058 & 0.996 & .0124039 & 0.443 & .0002451 & 0.988 \\
\hline Part time contracts after parental leave & -.0054081 & 0.751 & .0263559 & 0.270 & -.0177486 & 0.474 \\
\hline Working from home & .0130551 & 0.237 & -.0028842 & 0.846 & .0262108 & 0.108 \\
\hline Job sharing for leadership roles & -.016584 & 0.213 & .0543697 & 0.007 & -.0032154 & 0.864 \\
\hline Diversity trainings & .003429 & 0.791 & .0388907 & $0.042 * *$ & -.0109449 & 0.560 \\
\hline Mentoring & -.0076222 & 0.515 & .0309176 & $0.068 *$ & -.0111218 & 0.511 \\
\hline Personal coaching & -.001632 & 0.867 & -.0134462 & 0.324 & .009313 & 0.511 \\
\hline Company kindergarten & -.0037137 & 0.727 & -.0069902 & 0.634 & -.0125761 & 0.417 \\
\hline Support for child care financing & -.0069367 & 0.365 & -.0074758 & 0.476 & $7.79 \mathrm{e}-06$ & 0.999 \\
\hline Company counsel for family topics & .0128741 & 0.245 & -.0189138 & 0.217 & -.0113493 & 0.472 \\
\hline Quota for female managers & .0025474 & 0.762 & .010512 & 0.371 & .0165275 & 0.187 \\
\hline Conversations after return from parental leave & .0041103 & 0.666 & -.0137721 & 0.302 & .0116772 & 0.400 \\
\hline Targeted recruiting of women & .0022799 & 0.845 & -.044111 & $0.014 * *$ & -.0021091 & 0.901 \\
\hline Part time for mature staff & -.0038989 & 0.635 & -.0121725 & 0.290 & -.02956 & $0.022 * *$ \\
\hline Systematic development for mature staff & .0253908 & 0.248 & -.0083276 & 0.779 & .0370336 & 0.244 \\
\hline Horizontal moves for mature staff & .0056944 & 0.708 & .0268102 & 0.212 & -.0059141 & 0.788 \\
\hline Targeted design of mixed age teams & -.0044355 & 0.819 & -.0825927 & $0.007 * * *$ & -.0400334 & 0.166 \\
\hline Ergonomic design of working space & .0008351 & 0.935 & .0055442 & 0.696 & -.0120788 & 0.421 \\
\hline Design of content for mature staff & .0060264 & 0.718 & .0031501 & 0.891 & .0137835 & 0.570 \\
\hline Leisure/sports activities & -.0167795 & $0.092 * *$ & .0267772 & $0.055^{*}$ & .000224 & 0.987 \\
\hline Targeted external recruiting of mature staff & .0074276 & 0.676 & -.0377271 & 0.137 & -.0114311 & 0.656 \\
\hline Personal health counseling & .0090948 & 0.358 & -.013825 & 0.312 & .0204049 & 0.162 \\
\hline Life phase oriented personnel development & -.0087246 & 0.661 & -.0025314 & 0.926 & -.0039703 & 0.890 \\
\hline Targeted recruiting of foreign professionals & .0118811 & 0.520 & .0102302 & 0.687 & .0518949 & $0.065^{*}$ \\
\hline $\begin{array}{l}\text { Targeted recruiting of professionals and } \\
\text { management with migration background }\end{array}$ & -.0245627 & 0.414 & .0355118 & 0.392 & -.0645274 & 0.148 \\
\hline Targeted recruiting of trainees and graduates & .0102268 & 0.680 & .0203787 & 0.552 & .0462366 & 0.208 \\
\hline Targeted design of international teams & -.0121869 & 0.415 & -.0578124 & $0.011 * *$ & -.0547533 & $0.020 * *$ \\
\hline Global talent pools & .0089618 & 0.398 & .0100397 & 0.490 & .0159969 & 0.300 \\
\hline Trainings / Workshops with international focus & .0068322 & 0.580 & -.0291776 & 0.100 & .0099397 & 0.577 \\
\hline Mentoring for international staff & -.0145378 & 0.464 & .0316834 & 0.253 & .0095826 & 0.737 \\
\hline
\end{tabular}




\begin{tabular}{lllllll}
\hline & \multicolumn{2}{l}{$\begin{array}{l}\text { Model c Variable: } \\
\text { Embeddedness [var 346] }\end{array}$} & \multicolumn{2}{l}{$\begin{array}{l}\text { Model d Variable: } \\
\text { Commitment [var 347] }\end{array}$} & \multicolumn{2}{l}{$\begin{array}{l}\text { Model d Variable: Work life } \\
\text { balance [var 348] }\end{array}$} \\
\cline { 2 - 7 } & Beta & $\mathbf{P}$ & Beta & $\mathbf{P}$ & Beta & P \\
\hline Department to support foreign professionals & -.0013346 & 0.875 & -.0121072 & 0.309 & .0001941 & 0.987 \\
& 3.222547 & 0.000 & 3.341475 & 0.000 & 2.324853 & 0.000 \\
\hline
\end{tabular}

Finally, table 6 combines results from the employer and employee surveys, adding the mean results of the employee survey for specific organizations to the employer data set as dependent variable for the use of specific instruments. The dependent variables include overall diversity climate as perceived by employees in the organization (model a), personal preferences on career and performance (model b), personal ambition (model c) and overall perception of talent culture (model $\mathrm{d}$ ). The effect of diversity measures on the diversity climate are confirmed by this approach, while the use of diversity measures appear to have little to know effect on the other variables. Part time offers for mature staff seems to be the one measure with a positive effect on almost all variables.

Table 6. Results of regression analysis (employer survey): effects of the use of instruments on overall diversity perceptions by employees in the organization (model a), personal preferences on career and performance, the perception of talent culture (model c), and on personal ambition (model d).

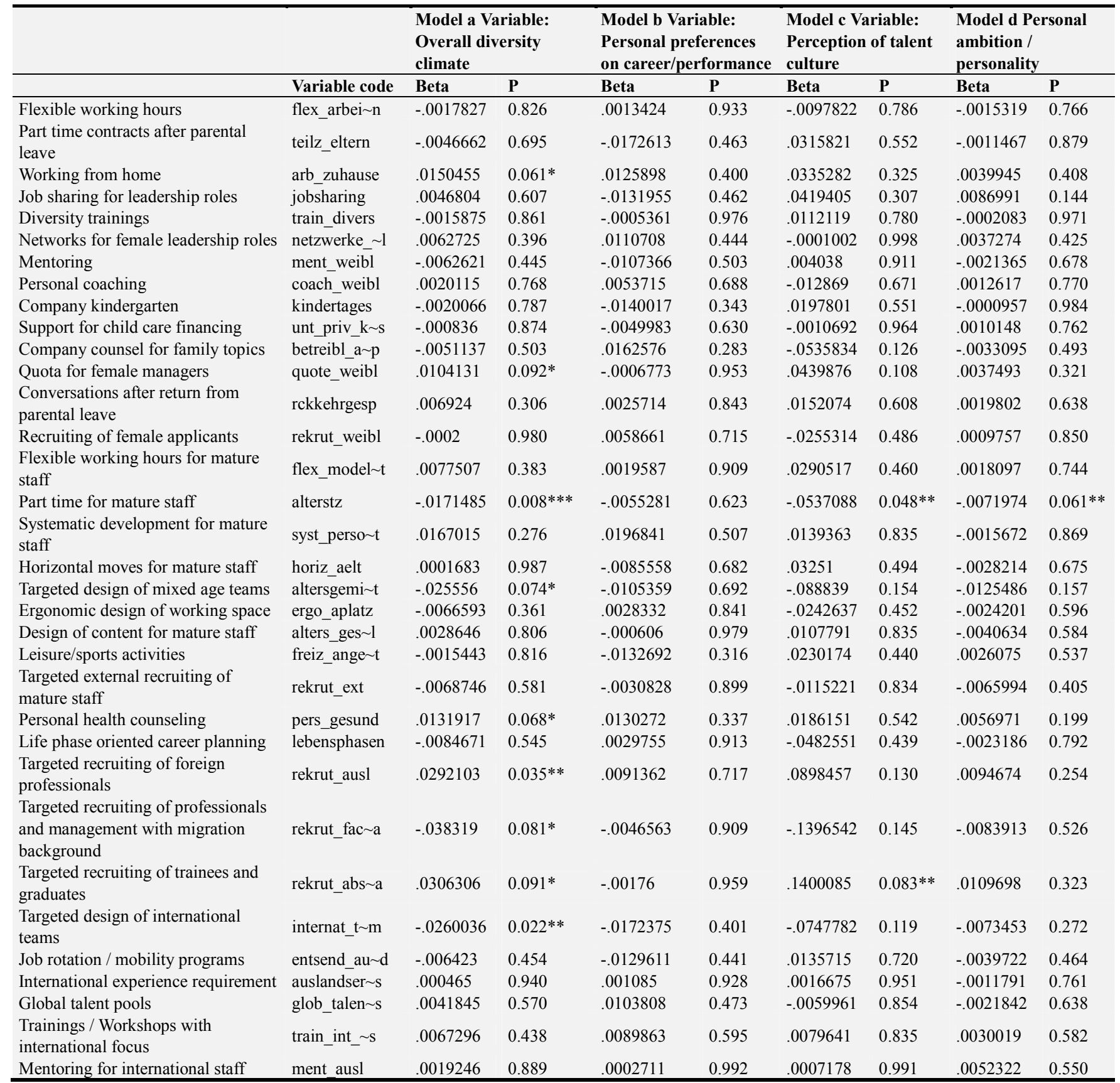




\begin{tabular}{llllllllll}
\hline & & \multicolumn{2}{l}{$\begin{array}{l}\text { Model a Variable: } \\
\text { Overall diversity } \\
\text { climate }\end{array}$} & $\begin{array}{l}\text { Model b Variable: } \\
\text { Personal preferences } \\
\text { on career/performance }\end{array}$ & $\begin{array}{l}\text { Model c Variable: } \begin{array}{l}\text { Perception of talent } \\
\text { culture }\end{array} \\
\text { Beta }\end{array}$ & $\begin{array}{l}\text { Model d Personal } \\
\text { ambition / } \\
\text { personality }\end{array}$ \\
\hline & Variable code & Beta & P & Pera & Beta & P & Beta & P \\
\hline Department to support foreign staff & abtl_unt_a a & .0018824 & 0.751 & .0029208 & 0.802 & -.0063401 & 0.810 & .0029295 & 0.439 \\
& cons & 2.960492 & 0.000 & 3.521957 & 0.000 & 2.919169 & 0.000 & 3.274776 & 0.000 \\
\hline
\end{tabular}

\section{Discussion}

The key finding of this study is that German organizations are applying too little diversity measures in their organizations and that if they do apply measures they are often applying not the most effective ones. This seems partly be due to a low assumed significance of general diversity demands, lower levels of societal and economic embeddedness of the topic, as well as lower levels of diversity infrastructure. It seems that despite experts know better, too often only the more traditional and state regulated measures with respect to gender diversity are being applied. For example, the HR experts in Germany prioritize gender quota as second most effective gender diversity instrument in German organizations, however only $27 \%$ of organizations apply this measure. It appears the organizations are waiting for the state to regulate diversity and then they are complying with it rather than actively creating a set of unique differentiating measures that can be used to attract and retain human capital which is what German organizations should be doing in order to remain successful. The study results could therefore be used as evidence for future state regulation of diversity measures in Germany.

Furthermore, the difference between actual usage of instruments in German organizations as opposed to the prioritization of experts in these organizations is being used as indicator for the maturity of this diversity area in German organizations. According to this indicator, gender diversity shows the highest level of maturity in German organizations. In this area of diversity, employees also associate a higher level of commitment with the organization they work for.

The results of the present study show that many companies focus on promoting gender diversity. It appears that a great opportunity is being missed in the cultural context of Germany: Instead of providing differentiating measures which can support and strengthen their employer branding, the companies focus on offering part time arrangements and more flexible working hours. Measures designed to promote age and ethnic diversity, in contrast, are used by less than one third of the responding companies. Overall, the results confirm that in many organizations in Germany diversity management appears to be shrunk to 'integrationism' with the main burden put to the member of the minority groups rather than using diversity as management tool (Tatli et al, 2007).

Increasing significance, but low levels of infrastructure

Only just under half (46 percent) of the respondents in the survey consider the significance of diversity management in their organizations to be "high" or "very high". However, 92 percent of the respondents stated that they expect the significance to increase or even greatly increase. It can be reasonably assumed that in Germany diversity will considerably gain in significance. It is therefore all the more important for companies to already now understand what diversity instruments are effective and what benefits such diversity measures could bring for their position in the "War for Talents".

Despite the relevance attached to the topic in almost half of the organizations, only 32 percent of the respondents stated that their organization has a Diversity Officer whose scope goes beyond the role of the Equal Opportunity Officer required under German law. In companies which have a Diversity Officer, this officer at least reports directly to the Management Board or to the division head in more than half of the responding organizations, while the rest reports to the department head.

Asked for the objectives of their diversity management efforts, 75 percent of the responding organizations stated they had defined objectives relating to gender management; considerably less have defined objectives relating to age and ethnic diversity (age diversity: 45 percent; ethnic diversity: 38 percent). Other diversity aspects (such as religion, skin color or sexuality etc.) were not covered in the survey because their lack of relevance had already been identified in the run-up to the study. Furthermore, the survey shows that in 57 percent of the organizations, the diversity topic is embedded in the HR strategy, but only 38 percent of the organizations have managed to also embed the topic in their corporate strategies - although the involvement of other diversity aspects would indeed be important to mitigate the increasing shortage of professional and management personnel, especially in what in Germany are called the "MINT" professions (mathematics / information technology / natural sciences / technology), e.g. by retaining older talents, attracting foreign talents and generally enhance the organization's attractiveness as an employer vis-à-vis the current workforce and potential candidates.

Prioritization and prevalence of gender diversity instruments in German organizations.

The use of gender diversity instruments differs from the prioritization through experts. This difference is being used as indicator for the maturity of diversity in Germany.

The main section of the questionnaire focused on the specific instruments used to increase diversity. Most of the instruments mentioned by the respondents are aimed at promoting female employees and at enhancing the compatibility of family and career. Figure 3 shows an overview of how many of the organizations consider the listed measures to be "widely available" (4) or "largely available" (5).

The most common instruments appear to be those which contribute to making work arrangements more flexible, e.g. "flexible working hours" or "part-time contracts after 
parental leave", while instruments that include financial support, such as "company kindergarten" or "support for private child care financing" are mentioned by less than 25 percent of the respondents. Thus instruments including financial support, such as "company kindergarten" and "financial support for private child care financing" are significantly less common than instruments designed to promote the compatibility of family and career.

Traditional programs for the advancement of women, such as mentoring, coaching or networks for female employees, play a role in less than one third of the responding organizations. It is surprising, however, that after all around 27 percent state that their company is implementing a "gender quota" policy to increase the number of women in leadership positions. Even though this instruments is being discussed very controversially in society in German at large, it appears to be relatively undisputed once implemented in practice. In light of the fact that German organizations appear to react predominately to state regulated diversity policy, the implementation of a gender quote in Germany should be reconsidered.

In order to measure the significance of the various instruments independent of their commonness, the survey participants were asked to prioritize the instruments by usefulness. This permits a comparison between actual practice and the perceived usefulness of the instruments on the part of the HR managers. Instruments which on average were considered to be relatively useful while their availability is still below average can thus be deemed to be topics that will gain in importance. The results are shown in a scatter plot (Fig. 3). Respondents had the option to assign top, secondary or tertiary priority to each instrument. In the next step, an average priority was calculated for each instrument, which could attain a maximum value of 3 (and a minimum of 0 for those instruments that were not on the priority list of any of the respondents). Of particular interest are the instruments in the left upper quadrant, which are not yet relatively common in practice but which are attributed a relatively high usefulness. For example: the instrument "job sharing" is widely or largely available in only 4.6 percent of the responding companies, while at the same time it ranks relatively high in terms of the priority attributed to it. This instrument might be an effective option for companies to distinguish themselves as attractive employers. HR managers also perceive the "quota for female managers" as a relatively useful instrument, which can likewise be interpreted as an option to enhance the company image. Support for child care by means of a company kindergarten may also be another topic of the future. Holding the top ranking by a wide margin, "flexible working hours" are the instrument HR managers consider to be the most effective one; however, as this instrument is already quite common in many organizations, it offers less potential for differentiation from other employers.

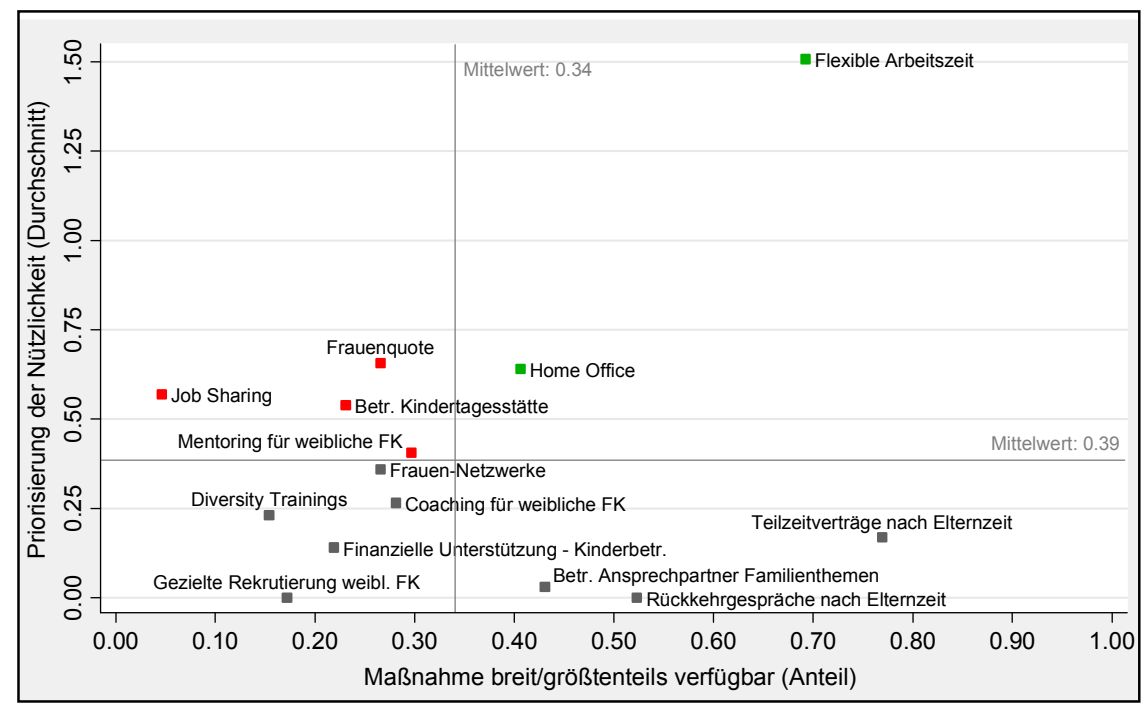

Figure 1. Use and prioritization of gender diversity instruments $(n=63)$.

Overall, it is surprising that organizations apply different measures than would be recommended by practicioners or HR experts which are colored red I the above chart. In addition, the instruments prioritized by HR experts appear to be preferred by employees. Thus, employees appear to be more progressive than what their organizations are willing to implement as their preferences are aligned with the prioritization of HR experts. Overall, however, with regards to gender diversity, many companies appear to have hit the mark with offering flexible working hours to their employees, because this instrument is actually associated with a significant difference in the outcome variable (higher proportion of women in the workforce of the organization).

Prioritization and prevalence of age diversity instruments in German organizations.

Age diversity is especially about retaining "older", more mature staff members. Figure 2 shows that the needs of the older generation are addressed especially by means of providing ergonomic design of working space, leisure/sports offers, and part-time arrangements (see the dots arranged along the X-axis). However, it are precisely these instruments which are attributed a relatively low usefulness. In contrast, the instruments "systematic development for mature staff", "design of content for mature staff", "horizontal moves for 
mature staff" and "targeted design of mixed age teams" are attributed a relatively high usefulness, although these instruments are today applied only in approx. 10 percent of the responding organizations. Therefore, the topics of "Systematic personnel development" and "Mixed age teams" can be considered as priority topics to come.

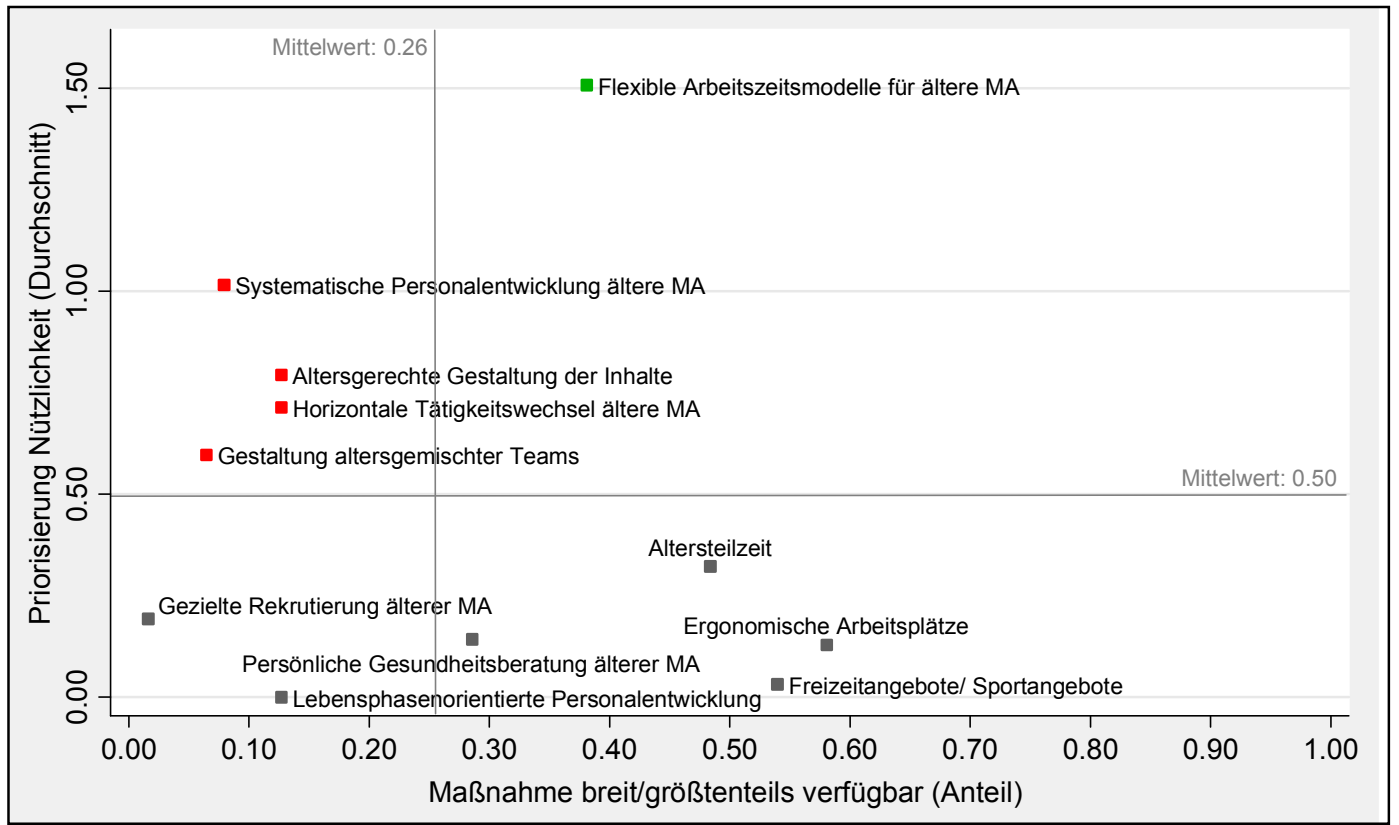

Figure 2. Actual use versus prioritization by HR experts of age diversity instruments.

The present study shows that specific needs of the older generation of employees are being primarily addressed by measures aimed at improving workplace ergonomics, health counseling, part-time arrangements for mature staff or leisure/sports offers. Qualitative topics, such as systematic HR development for mature staff, targeted design of mixed age teams and especially recruiting of mature staff play a secondary role only. Having in mind that this is where HR officers place a special focus on, this makes clear that there is a huge gap between intention and reality. Nevertheless, every company today faces the question of which value added can be generated bat he targeted use of instruments to enhance age diversity. A look at the detailed results of the survey makes clear that the proportion of employees aged between 56 and 65 was higher in those organizations that do apply instruments such as personal health counseling or mixed age teams than in those who do not apply such instruments. HR officers consider the instruments currently used by the companies - such as improvement of workplace ergonomics, leisure/sports offers and part-time arrangements for mature staff - to have relatively little usefulness. On contrast, systematic HR development for mature staff, design of content for mature staff, horizontal moves for mature staff and targeted design of mixed age teams are attributed a relatively high usefulness, although these instruments are today applied only in approx. 10 percent of the responding organizations. Interestingly, these measures are also prioritized by employees. The most effective age diversity measures are currently not particularly widely used but they are at least attributed a relatively high priority by HR officers and will therefore move more in the focus. In future, more targeted development for mature staff or opportunities for job rotation could be offered for mature staff as these are also prioritized by employees. Therefore, the evidence in the area of age diversity differs from gender diversity. Employers prefer instruments which leverage workforce flexibility, while HR experts seem to prioritize instruments which actually make jobs for the elderly more attractive and can therefore provide a more long term economic benefit. As the employees themselves also would prefer these instruments, German organizations currently don't seem to take advantage of the chance for differentiation using age diversity measures. Thus, the chance for differentiation in employer attractiveness is currently not being used.

Prioritization and prevalence of ethnic diversity instruments in German organizations.

Finally, in the area of ethnic diversity, German organizations actually seem to have the biggest issue. At the same time, this area could offer the largest opportunity for differentiation. Organizations consider "deployment of management personnel to jobs abroad", "targeted recruiting of foreign professional/management personnel", and "global talent pools" to be effective measures to increase the proportion of non-German staff. In addition, the "targeted recruiting of professional/management personnel with a migrant background" - which is not currently very common - is also considered to be relatively effective; this is understandable given the fact that organizations can avail themselves of ethnic diversity at relatively low cost while the deployment of German staff abroad is considerably more expensive. Covering internationality by deploying German staff with international experience seems therefore to be a topic which will increase in significance in the years to come. 


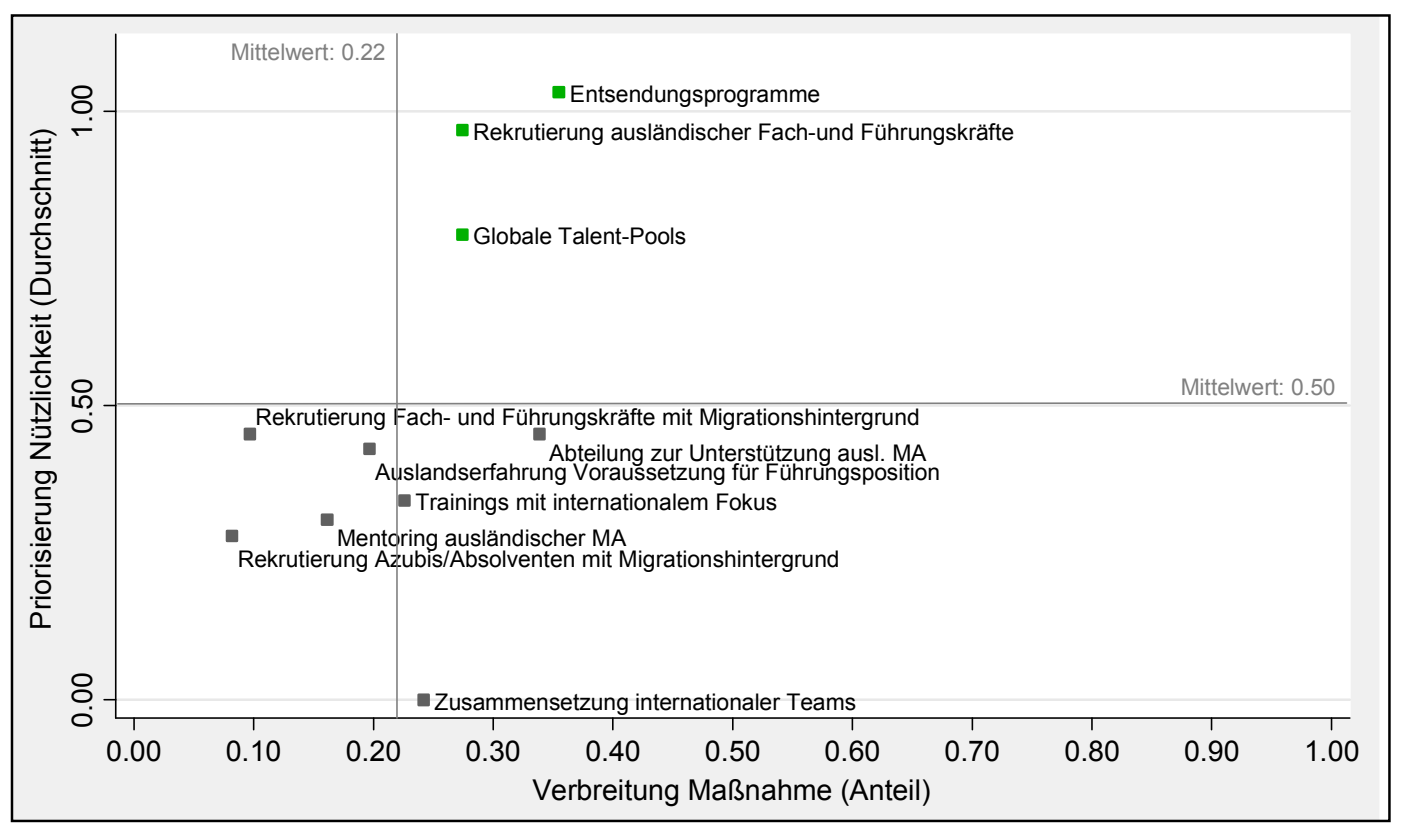

Figure 3. Actual use versus prioritization by HR experts of ethnic diversity instruments.

Organizations consider both, deployment of executive personnel to positions abroad and targeted recruitment of internationally experienced staff to be important measures. However, there is awareness of the differences between these two instruments, which is reflected by the fact that targeted recruiting is given priority. By pursuing this approach, the organizations miss out on specific cultural knowledge and knowledge of local customs and habits. Furthermore, the deployment of German staff and the training measures involved have proven to be a rather costly exercise. So why not enhance ethnic diversity within the company and harness the potentials inherent therein? Recruitment of professional and management personnel and (younger) trainees with migration background is increasingly seen as a way to move to the future. This intention is also indicated by the correlation matrix with the proportion of non-German staff members as dependent variable.

Companies consider the deployment of executive personnel to positions abroad, targeted recruitment of foreign professional/management personnel and global talent pools to be effective measures for increasing the proportion of nonGerman employees.

In addition, the focused recruiting of professional and management personnel with migration background - an instrument currently not very widely used - is considered to be relatively effective; this is understandable given the fact that organizations can avail themselves of ethnic diversity at relatively low cost while the deployment of German staff abroad is considerably more expensive.

However, in general it can be said that, as far as ethnic diversity is concerned, German organizations do not yet appear to have "a good nose": none of the particularly effective measures is currently very common in German organizations, nor do HR managers perceive them as particularly useful. Bringing internationality into the company through German staff with experience from working abroad could be replaced by hiring more international staff in the home country.

Quantitative (measurable) effects of the use of diversity measures on key metrics.

The analysis of effectiveness of diversity measures provides insights in the actual practice in the organizations in Germany. In order to gain insights into the actual effectiveness of diversity measures on different outcome variables, regression analyses were done in a next step, with the specific instruments being the explanatory variables. Tables 3, 4 and 5 show the results of the analyses. The proportion of female employees in the overall workforce and the proportion of female managers were used as outcome variables for gender diversity; the proportion of mature staff was used as outcome variable for age diversity and the proportion of non-German staff was used for ethnic diversity. In the regression analysis, various influencing parameters, such as industry, organization size and number of used diversity instruments in each organization were controlled in order to isolate the effect as much as possible. The analysis shows which measures have a significant impact on the outcome variables and which don't (Figure 4). However, given the small number of observations covered, results should be interpreted with some caution. On the basis of the analysis results, the differences in the depth of experience of the organizations with the various diversity aspects can be considered to be confirmed. As regards the gender aspect, many companies appear to have hit the mark with offering flexible working hours to their employees, because this instrument is actually associated with a significant difference in the outcome variable (higher proportion of women in the workforce of the organization). In the case of age diversity, the situation is different in that the most effective instrument in this field is not particularly common but given priority by 
HR managers and that therefore quite some focus will be placed on this instrument in future. As far as ethnic diversity is concerned, German organizations do not yet appear to have

\begin{tabular}{|c|c|c|}
\hline $\begin{array}{l}\text { Diversity } \\
\text { Dimension }\end{array}$ & Outcome variable & $\begin{array}{l}\text { Effective instrument (making a significant } \\
\text { difference in the outcome variable) }\end{array}$ \\
\hline $\begin{array}{l}\text { Gender } \\
\text { Diversity }\end{array}$ & $\begin{array}{l}\text { Proportion of female employees } \\
\text { in overall workforce }\end{array}$ & $>$ Flexible working hours \\
\hline & $\begin{array}{l}\text { Proportion of female employees } \\
\text { on top } 3 \text { management levels }\end{array}$ & $\begin{array}{l}>\text { Conversations after return from parental } \\
\text { leave }\end{array}$ \\
\hline Age Diversity & $\begin{array}{l}\text { Proportion of mature employees } \\
\text { (age }>56)\end{array}$ & $>$ Horizontal moves for mature staff \\
\hline $\begin{array}{l}\text { Ethnic } \\
\text { Diversity }\end{array}$ & $\begin{array}{l}\text { Proportion of non-German } \\
\text { employees }\end{array}$ & $\begin{array}{l}>\text { Targeted recruiting of foreign } \\
\text { professionals/management } \\
>\text { Recruiting of professionals and } \\
\text { management with migration background } \\
>\text { Targeted design of international teams }\end{array}$ \\
\hline
\end{tabular}

Figure 4. Instruments making a significant difference in the outcome variable (in companies applying the respective instruments).

Due to the potential benefits and possible state regulation in Germany, the topic is still being discussed widely. The discussion is also still controversial and has a political dimension. However, it is surprising that the levels of diversity measures are so low. In this context it appears that in most cases only the aspect of equal opportunities for women is being discussed, whereas mature employees and people with a migration background are being neglected in the debate. For employees, their decision in favor of or against a job offer depends increasingly on a multitude of different factors involving aspects of diversity management. In this regard, such aspects as job satisfaction and corporate/business policy of the company play an ever increasing role.

The issue of diversity management continues to be a very topical one, not least due to the ongoing discussion on the pros and cons of a mandatory quota of women in management positions. However, the results confirm that Germany is still very much behind due negative experience with migration, ethnic diversity, and a culture of inclusion. Companies in Germany would be well advised to move strategically and turn the weakness and other countries mistakes into advantage.

In synthesis, the results of Table 4 and 5 show the complication of diversity management. Table 4 shows that employees associate a positive diversity and inclusion climate as well as high levels of work live balance with the usage of diversity instruments. Table 5 shows the different priorities of employers. In this case, the high usage of instruments is associated with improved career opportunities and improved commitment to manage fluctuation. It is critical for the success of diversity measures to integrate the different perspectives and find the tools that satisfy the needs of employers and employees. As Table 4 shows, from an employee perspective it is mostly the overall perception on diversity practice and discrimination as well as perception on "a good nose": none of the particularly effective measures is currently very common in German organizations, nor do HR managers perceive them as particularly useful.

Effective instrument (making a significant difference in the outcome variable

work live balance which is impacted by the use of diversity management (gender, age, and ethnic) in the organization. In contrast, table 5 shows that from an employer perspective it is the perception of career opportunities and commitment to the organizations which is impacted by the use of diversity measures. The picture evolved that the personal character of the workforce is important as it determines what is preferred by employees. Therefore, the culture of the workforce becomes an important factor. The success of diversity measures is higher the more socially aware the workforce is. This means, the diversity instruments have a higher efficacy in the case of a workforce with higher social value orientation.

Qualitative (measurable) effects associated with the use of diversity instruments.

The results of the employee survey in table 4 show on average a positive effect of the use of diversity instruments on the overall diversity climate and the personal perception of work life balance, as well as a negative effect on discrimination. The results of the employer survey in table 5 show a positive effect on career opportunities as well as employee commitment. The different results speak to the different perspectives, expectations and priorities of employees and employers in organizations around the use of diversity instruments which are reflected by the study results. Employers appear to appreciate employee development and commitment, whereas employees prioritize intuitively a positive atmosphere against discrimination as well as work life balance. The latter prioritization may not be identical with all employees, thus the effects of diversity measures may also be connected to the personality of employees. Thus personality of employees may determine success of diversity culture. The picture evolves that the personal character of the workforce is important as it determines internal preferences and perceptions on diversity. There are no statistical differences in the personality of employees with respect to 
the effectiveness of specific measures. However it does determine how positive instruments in can contribute to an atmosphere of inclusion in the organization. Personality determines how strong someone react positively to the culture of the organization, thus SVO and JS are important concepts in diversity management.

If employees personality is a differentiator for the success of diversity measures, organizations will need to attempt profiling the personality of employees (to the extend this is possible by corporate law). One appropriate key metric is social value orientation, which appears to be a valid differentiator for workforce perception. Social value orientation may determine which instruments are success critical and which are not. Personality may not determine which instruments are success critical but it determines the culture of an organization and if the culture is conducive to tolerance or not, which can be defined as intervening variable and will be described in the next section.

While employee personalities appear to be a determining factor of the success of diversity measures, corporate culture seems to be another factor. Examples of a talent culture may be to focus more on potential than on past performance. This has several reasons. Some of them are obvious, as people like to be faced with possibilities rather than with facts, as they imply the opportunities of improvement. Another reason is that often career advancements involve very different jobs and requirements and that often people simply won't perform as well on the new job than on the previous job. Therefore it is recommended to focus in talent management more on potential future than on past performance, and to focus on potential assessment rather than evaluation?

Therefore, research shows that talent management tools are more effective if they are combined with personality assessing instruments gauging people's potential. The reason is that it is much more empowering to an individual to be assessed on the basis of his/her potential than on the basis of past performance. On the other hand, the level of transparency is lower if past performance is not evaluated. People are largely being assessed by the past performance, yet research shows potential should be by far the larger driver for assessments. But if this is the case you need to consider personality.

If it is so important to include the personality of individuals in the design of talent management instruments, then it is suggested to include the most current personality groupings available. In previous research other qualitative success factors of talent management was shown as selfcontrol (Landwehr 2016).

Intervening variables on diversity management success

The results suggest that the perception of diversity climate, discrimination as well as work life balance are intervening factors for diversity measure success. Moe precisely, the results suggest that employees with higher levels of social value orientation prefer those diversity instruments over other instruments which are actually more effective with respective to objective metrics and could also be more effective for differentiation in the context of employer branding. One of the personality traits which reflects how people evaluate outcomes for themselves and others is 'social value orientation'. It is regarded as a personality trait and has been identified as an important determinant of cooperative motives, strategies, and choice behavior (Kollock 1998), and is therefore relevant for perception of diversity measures. The literature generally differentiates among prosocials, who tend to maximize outcomes for both themselves and others (i.e. cooperation) and to minimize differences between outcomes for themselves and others (i.e. equality); individualists tend to maximize their own outcomes with little or no regard for others' outcomes; and competitors tend to maximize their own outcomes relative to others' outcomes, seeking relative advantage over others (Van Lange, 1997). Based on the results, high SVO is associated with a higher diversity culture.

De Dreu \& Boles (1998) have linked SVO to negotiation heuristics expressing a specific perspective on life and thus explained that SVO is linked to moral convictions. Similarly, the integrative Model of Social value orientation by Van Lange (1999) argues that a prosocial orientation should be understood in terms of enhancing both joint outcomes and equality of outcomes.

From the beginning of SVO research, game theoretical experiments have been the basis for tracking the outcome distribution of certain behavior patterns. Accordingly, Kuhlman \& Marshello 1975, Sattler \& Kerr 1991, according to Lange et al. (1997) have pitted the results of cooperation versus non-cooperation of prosocial, individualistic and competitive preferences. Not surprisingly, a prosocial value disposition leads to higher returns, and therefore prosocial behavior would clearly be preferred over other behavior patterns in most situations. In addition, there are some selfenforcing factors to the positive effect of prosocialism, i.e. the positive impression on others (Iedema and Poppe, 1996). These factors need to be taken into account when making assumptions about the value of a prosocial value orientation and the effect of diversity instruments.

The concept of justice sensitivity aims at explaining interindividual differences in reacting to unfair situations (such as discrimination) and was first described by Schmitt et al. (1995) as sensitivity to befallen injustice. Based on this concept, Schmitt \& Mohiyeddini 1996 suggested four different indicators for a person's sensitivity to befallen injustice, i.e. (a) frequency of perceived injustice, (b) intensity of anger, (c) intrusiveness $\sim$ perseverance of thoughts about the event, and (d) punitivity. These indicators resulted in the definition of a specific perception pattern of justice sensitivity for which they identified distinct inter-individual differences. In order to improve the predictive power of the model, Fetchenhauer and Huang (2004) differentiated the personality trait of justice sensitivity into three subdimensions: sensitivity with regard to experiencing injustice towards oneself (JSvictim); sensitivity to observing that others are treated unfairly (JSobserver) and sensitivity to profiting from unfair events (JSbeneficiary). These dimensions were successfully used to predict decision 
making in a number of game theoretical paradigms. Therefore, performance management instruments should include components of a personality regulation which means that performance management takes personality into account.

Finally, work-life balance seems to an intervening variable for the success of diversity instruments as well. As a result, if companies finally succeed in recruiting qualified employees and managers, this will have a decisive influence on their competitive position. When making their decision on which job they choose and what sacrifices they are ready to bring, candidates consider family aspects and their desire to have their lives not solely be determined by their work. Exciting tasks, personal development opportunities and the corporate/business policies of a company are therefore in most cases more important than lucrative pay at renowned companies.

Diversity must become established as a central guiding principle in companies and understood as a factor that positively impacts company success.

Thus, the study makes clear once again that although companies do have initial ideas regarding diversity and talent management, they will need to put into place further measures. Nevertheless, as regards the study conducted by the University of Cologne it can be regarded as critical that no consistent definition of "high potentials" can be found. Furthermore, it is not clear whether an increased proportion of employees belonging to the more mature generation is desirable at all. After all, this generation is often considered to be associated with lack of IT knowledge, excessive salaries or susceptibility to age-related diseases. The study does not permit any conclusions as regards this topic and the different definitions of "high potentials" the individual HR managers have in their minds.

However, it is beyond doubt that in the coming years the overall topic of talent and diversity management will continue to grow in importance and that interesting innovations will be seen in this field. Companies must face up to the challenges of the labor market and take suitable, individual actions to secure corporate success.

In the context of talent and diversity management, social media is becoming more and more important, as diversity compentencies are being shared in social media. Therefore, the social media assessment is turning into an important intervening variable for diversity success as well.

Likewise, as a general trend it was found that social media portals are becoming more important and that especially the younger generation prefers using the web over conventional job advertisements. It is therefore important for the companies how to establish and design their web presence to attract high potential candidates. However, it is not always necessary to look for talents outside of the company. According to the HR managers responding in the survey, structured potential assessments, internal job portals and talent review workshops are indeed effective and common measures for identifying the leaders of tomorrow. At the same time, however, the study has also revealed a lack of talent pools and $360^{\circ}$ feedback rounds. This can certainly be attributed to the effort and cost involved in keeping the individual employee profiles up to date. However, each individual company has to make its own decision on whether the costs thereby incurred exceed the worth of the positive results. Without doubt, communication is another aspect which is neglected in many companies and, as a result, talents available in one department often remain unknown to the other departments or divisions of an organization. When it comes to an effective selection of talents and to actions to advance these talents, many companies fail due to lacking follow-up on promotion promised to the employees (65 percent) or because there are no career prospects at all (76 percent). This illustrates that in many cases the companies themselves are responsible for their lack of suitable junior managers.

In addition, in the first instance, many companies are facing the question about the form of talent performance measurement and how and to what extent it is possible to identify differences to non-potentials. For performance assessment, the surveyed HR managers primarily use the instruments of a fixed scale, separation of performance and potentials, and specific agreements on objectives. "Forced" measures or conferences with several managers, in contrast, are less appreciated, firstly because of the time and cost effort involved, and secondly because forced measures are generally considered counterproductive. However, as regards performance assessment, top priority should be given to transparency for the employee. Only by treating employees in a consistent manner and in the absence of any arbitrariness can employees be motivated and triggered to perform better. At the end of the day, each and every employee will in the medium and long run face the question about which personal development prospect is provided by his or her company. For employees, such aspects like the opportunity to take on more responsibility or to get involved in an international project are often important criteria for a change of employer. In this regard, the study conducted by the Seminar for Human Resource Management at the University of Cologne identified focused measures, such as personal development plans, stretch assignments, portals for training and continued professional development and special development programs for high potentials as a priority. It is the aim of HR managers to offer individual opportunities and incentives for talents. However, promotion measures are limited to such employees who show personal ambition and a will to develop further. Nevertheless, the study shows that there are indeed personnel promotion opportunities, which are, however, in most cases only accessible for high potentials. Due to this, talents on the lower levels, which are of great importance to the company, take a back seat. It is therefore necessary to find ways to offer high potentials opportunities to develop their skills while at the same time providing suitable measures for talented employees even if they are not high potentials. In order to avoid loosing qualified employees, most companies will have to invest in this field.

To end, organization should dare more, and acknowledge that they can be the drove of change. If there use diversity as differentiator, they will maximize their benefits. 


\section{Summary of Results}

Although the topic of diversity does not rank at the top of the agenda in many companies in Germany, the interest of HR experts in the topic appears to increase. In many companies, the primary focus is still on the gender aspect but his might change soon as the demographic change becomes more tangible and employees will increasingly have to rely on employees with a migration background and on more mature employees.

Our analysis indicates that some measures are perceived to be particularly useful although they are not yet widely applied. This includes the application of a quota of women in management, company kindergarten, job sharing and systematic personnel development for mature employees. Companies should therefore consider applying such measures in practice, not only to make use of the potential available in the labor market but also to distinguish themselves from competitors in the labor market. For quite some time now, ratings of the quality of companies as employers have become widespread in the Internet and the approach of companies to diversity has become an important rating criterion for many users.

\section{Recommended Actions for Companies}

- To improve diversity, assess effective measures in a focused manner: If a company wants to improve promotion of workforce diversity, also to distinguish itself as an employer, the measures analyzed in the present study can provide important leads. Especially those measures that are not yet widely used but assessed as relatively useful (red) should be considered in the discussions.

- Analyze the effectiveness of the company's offers to encourage diversity (internal analysis): A survey among the company's own employees about the effectiveness of the various measures could probably produce the most meaningful findings as regards the usefulness of the measures. This could be implemented without much effort by including suitable questions in employee surveys that are carried out anyway. The assessment of the measures by the target group itself will firstly ensure more objectivity of the assessment and secondly provide the management with direct leads as to how the measures should be developed further.

- Gear the company's offers to the future personnel requirements (external analysis): Companies should understand offers to encourage diversity as a necessity for finding and retaining suitable personnel in the future. It is important to understand in which areas, professional groups and functions a personnel shortage may emerge in the company within the near future (five years). This shortage/demand must then be compared to the supply of suitable resources available in the labor market. Depending on the identified sources of professionals (e.g. mature employees or professionals from abroad) the company could tap, it will then be possible for companies to focus on and expand specific diversity encouraging offers. Leads as to which measures are useful can be derived from studies or from findings arrived at in the company itself.

The results of the present study do not cover employee perception. Suitable additional analyses could perhaps produce more objective assessments of the success of the measures. Thus, a second phase of the study is planned to survey the employees of the responding companies for their perception of the measures offered and the effectiveness of such measures. The aggregated data can then be analyzed in combination.

The results of the present study show HR managers' assessment of the effectiveness of specific measures in practice, and the actual success of the measures. In contrast, the current debate on some specific diversity measures, such as "Mandatory quota for female managers", "EU Blue Card" or "anonymized job applications" are often based on key figures, rules and regulations instead of an analysis of the objective, economic and social benefits of having a diverse workforce. A better balance with scientific examination of the benefits of and challenges involved in diversity could greatly contribute to both the debate at large and the practicability of methods applied and approaches taken.

\section{References}

[1] Anfuso, D., (1997), Diversity keeps up with the times, in: Personnel Journal 74 (7), p. 30-38.

[2] Arrendoro, P., Successful Diversity Management Initiatives (1996).

[3] Briscoe, J. P., \& Hall, D. T. (2005). Protean and boundaryless career assessment collection. Boston, MA: Unpublished, copyrighted scale collection.

[4] Capowski, G (1996), Managing diversity, in: Management Review 86 (6), pp.12-20.

[5] Carnevale, A. and S. Stone (1994), Diversity beyond the goler rule, in: training \& Development 48 (10), pp. 20-40.

[6] Chen, Z. X. and Aryee, S. (2007) 'Delegation and Employee Work Outcomes: An Examination of the Cultural Context of Mediating Processes in China', Academy of Management Journal, Vol.50, No.1 pp.226-238.

[7] Choudhury, E. H. (1996), The nature and significance of workforce diversity: Orientations of Sate and Urban Administrators, in: International Journal of Public Administration 19 (3), pp. 399-423.

[8] Colarelli, S., Bishop, R., Career Commitment, in: Group \& Organization Studies; Jun. 90, Vol. 15 Issue 2, p 158-176, 19p.

[9] Collings, D. G. and Mellahi, K. (2009), 'Strategic Talent Management: A review and research agenda,' in: Human Resource Management Review, 19: 4, 304-313.

[10] Costea, B., Introna, L. (2008), On the mystery of the other and diversity management, in: Phenomenology, Organization and Technology, Lisbon, Universitade Catolica Editora and POT Books. 
[11] Cox, T. (1994), A comment on the language of diversity, in: Organizations 1 (1), pp. 51-57

[12] De Dreu, C. K. W and Boles, T. (1998). Share and share alike or winner takes all? Impact of social value orientation on the choice and recall of decision heuristics in negotiation. Organizational Behavior and Human Decision Processes, 76.

[13] D'Souza, D. (1997), The diversity trap: usefulness of cultural diversity training by corporations, in: Forbes $159 / 2)$, pp.8384 .

[14] Elias, C. and Sonnenfeld, J. L. (1994), Divere approaches to managing diversity, in: Human Resource Management 33(1), pp.79-109.

[15] Fetchenhauer, D., \& Huang, X. (2004). Justice sensitivity and behavior in experimental games. Personality and Individual Differences, 36, 1015-1031.

[16] Garnsey, E. and Rees, B. (1996). Discourse and enactment: Gender inequality in text and context. Human Relations, 49 (8): 1041-1064.

[17] Humphries, M., Grice., S., (1995), Equal employment opportunity and the management of diversity: A global discourse of assimilation?, in: Journal of Organizational Change Management, Vol. 8 Iss: 5, pp.17-32.

[18] Iedema, J. and Poppe, M. (1996), 'The Effect of Selfpresentation on Social Value Orientation', Journal of Psychology, vol. 134.

[19] Johnson, W. B., Workforce 2000: Work and Workers for the $21^{\text {st }}$ Century, Hudson Institute (1987).

[20] Jonsen, K., et al., (2013), The tragedy of the uncommons: Reframing workplace diversity, in: human relations, 66 (2), pp. 271-294.

[21] Kersten, A. (2000) "Diversity management: Dialogue, dialectics and diversion", Journal of Organizational Change Management, Vol. 13 Iss: 3.

[22] Kandola, R. S., Fullerton, J., Diversity in Action: managing the mosaic, CIPD Publishing (1998).

[23] Kollock, P., (1998b): Social Dilemmas. The Anatomy of Cooperation; in: Annual Review of Sociology.

[24] Konovsky, M. A., \& Cropanzano, R. (1991). Perceived fairness of employee drug testing as a predictor of employee attitudes and job performance. Journal of Applied Psychology, 76, 698-707.

[25] Köppel, P., Yan, J., Lüdicke, J., (2007), Cultural Diversity Management in Deutschland hinkt hinter her, Bertelsmann Stiftung, Gütersloh.

[26] Krell, G., (2008), Diversity Management: Chancengleichheit für alle und auch als Wettbewerbsfaktor, in: Chancengleichheit durch Personalpolitik, Gabler, 5. Aufl. Wiesbaden, S. 63-80.

[27] Kuhlmann, D. M. \& Marshello, A. (1975). Individual differences in game motivation as moderators of preprogrammed strategic effects in prisoner's dilemma. Journal of Personality and Social Psychology, 32, 922-931.

[28] Landwehr, J. (2016), The Principle of 'Self-Control' in the Design of Instruments, Processes and Procedures: True Success Factors of Talent Management in Journal of Human Resource Management.
[29] Lewis, R. E., \& R. J. Heckman (2006), 'Talent management: A critical review,' in Human Resource Management Review, 16: $139-154$.

[30] Lobiecki, A., Jack, G., Critical Turn in the Evolution of Diversity Management, British Journal of Management, Vol. 11, Special Issue, S17-S31 (2000).

[31] McDonald, D. M. (2010). The evolution of 'diversity management' in the USA: Social contexts, managerial motives and theoretical approaches. Institute of Business Research, Daito Bunka University.

[32] Mobley, M. and Payne, T. (1992), Backlash: the challenge to diversity training, in: Training and Development 46(12), pp. 45-52.

[33] Nemetz, P. L. and Christensen, S. L., The challenge of cultural diversity: Harnessing a diversity of views to understand multiculturism, in: Academy ogf Management Review 21 (2), pp. 434-462.

[34] Ng, T. W. H., \& Feldman, D. C. (2009). Age, work experience, and the psychological contract. Journal of Organizational Behavior, 30, 1053-1075.

[35] Nkomo, S., Cox, T. (1996). Diverse identities in organizations. In Handbook of organization studies, ed. S. Clegg, C. Hardy and W. Nord, 338-356. London and Thousand Oaks: Sage Publications.

[36] Lewis, R. E., \& R. J. Heckman (2006), 'Talent management: A critical review,' in Human Resource Management Review, 16: 139-154.

[37] Noe, R. A. (1996), "Is career management related to employee development and performance?", Journal of Organizational Behavior, Vol. 17, pp. 119-23.

[38] Noe, R. A., Noe, A. W., \& Bachhuber, J. A. (1990). Correlates of career motivation. Journal of Vocational Behavior, 37, 340 356.

[39] Ozbilgin, M. F., Tatli, A (2008), Global Diversity Management: An Evidence based approach, 2008.

[40] Peng, J.-C., Chui, S., F., An Integrative Model Linking Feedback Environment and Organizational Citizenship Behavior, in: The Journal of Social Psychology, 2010, 150 (6), 582-607.

[41] Robinson, C., Fetters, R., Riester, D., Bracco, A., The Paradox of Potential: A Suggestion for Guiding Talent Management Discussions in Organizations, in: Industrial \& Organizational Psychology; Dec2009, Vol. 2 Issue 4, p413-415.

[42] Robinson, Carl; Fetters, Rachel; Riester, Devon; Bracco, Anna (2009), 'The Paradox of Potential: A Suggestion for Guiding Talent Management Discussions in Organizations', Industrial \& Organizational Psychology, Dec2009, Vol. 2 Issue 4, p413-415.

[43] Roznowski, Mary (1989). Examination of the measurement properties of the Job descriptive index with experimental items, in: Journal of Applied Psychology, 74, 805-814.

[44] Sattler, D. N. and N. L. Kerr (1991) 'Migh versus morality explored: motivational and cognitive bases for social motives', Journal of Personality and Social Psychology 60 (5), 756-65.

[45] Schmitt, M., Neumann, R. \& Montada, L. (1995). Dispositional sensitivity to befallen injustice. Social Justice Research, 8, 385-407. 
[46] Schmitt, M., \& Mohiyeddini, C. (1996). Sensitivity to befallen injustice and reactions to a real-life-disadvantage. Social Justice Research, 9, 223-238.

[47] Seligman, D. (1994), Thinking about the gauntlet: diversity training programmes for federal agency employees are coercive, in: Fortune 130 (8), pp. 214-215.

[48] Smith, B. (1991), Diversity with a difference, in: HR Focus 68 (12), pp. 5-6.

[49] Subeliani, D., Tsogas, G., Managing diversity in the Netherlands: A case study at Rabobank, in: International Journal of Human Resource Management 16: 5 May 2005, pp 831-851.

[50] Suess, S., Kleiner, M., (2005; Diversity management in Deutschland, Ergebnisse einer Unternehmensbefragung.
[51] Tatli, A., at al., (2012), The role of regulatory and temporal context in the construction of diversity discourses: the case of the UK, France and Germany, in: European Journal of Industrial Relations 18 (4), pp. 293-308.

[52] Thomas, R., (1995), 'A Diversity Framework', in: Chemers, M. M., Oskamp, S., Costanzo, M. A. (eds.), Diversity in organizations: new Perspectives for a Changing Workplace, Thousand Oaks (Sage), pp. 306-317.

[53] Thomas, D. A., and Ely, R. J., (1996), Making differences matter: a new paradigm for diversity management, in: Harvard Business Review, Sept-Oct, pp. 79-90.

[54] Van Lange, P. A. M. (1999). The pursuit ofjoint outcomes and equality in outcomes: An integrative model of social value orientation. Journal of Personality and Social Psychology, 77, 337-349. 